\title{
The emergence of localized vortex-wave interaction states in plane Couette flow
}

\author{
By Kengo Deguchi ${ }^{1}$, Philip Hall ${ }^{2}$ and Andrew Walton ${ }^{3}$ \\ ${ }^{1}$ Department of Aeronautics and Astronautics, Graduate School of Engineering, \\ Kyoto University, Kyoto, 606-8501, JAPAN \\ ${ }^{2,3}$ Department of Mathematics, Imperial College London, \\ South Kensington Campus, London SW7 2AZ, UK
}

\begin{abstract}
The recently understood relationship between high Reynolds number vortex-wave interaction theory and computationally-generated self-sustaining processes provides a possible route to an understanding of some of the underlying structures of fully turbulent flows. Here vortex-wave interaction theory, which we now refer to as VWI, is used in the long streamwise wavelength limit to continue the development found at order one wavelengths by Hall and Sherwin (2010). The asymptotic description given reduces the Navier-Stokes equations to the so-called boundary-region equations for which we find equilibrium states describing the change in the VWI as the wavelength of the wave increases from $O(h)$ to $O(R h)$ where $R$ is the Reynolds number and $2 h$ is the depth of the channel. The reduced equations do not include the streamwise pressure gradient of the perturbation or the effect of streamwise diffusion of the wave-vortex states. The solutions we calculate have an asymptotic error proportional to $R^{-2}$ when compared to the full Navier-Stokes equations. The results found correspond to the minimum drag configuration for VWI states and might therefore be of relevance to the control of turbulent flows. The key feature of the new states discussed here is the thickening of the critical layer structure associated with the wave part of the flow to completely fill the channel so that the roll part of the flow is driven throughout the flow rather than as in Hall and Sherwin (2010) as a stress discontinuity across the critical layer. We identify a critical streamwise wavenumber scaling which when approached causes the flow to localise and take on similarities with computationally-generated or experimentally-observed turbulent spots. In effect the identification of this critical wavenumber for a given value of the assumed high Reynolds number fixes a minimum box length necessary for the emergence of localized structures. Whereas nonlinear equilibrium states of the Navier-Stokes equations are thought to form a backbone on which turbulent flows hang, our results suggest that the localized states found here might play a related role for turbulent spots.
\end{abstract}

\section{Introduction}

Our concern is with the structure of large amplitude modifications to shear flows. Here we concentrate on plane Couette flow, which of course is well-known to be linearly stable for all Reynolds numbers, but the structures we describe are generic and are applicable to a wide range of shear flows, including those which are linearly unstable. In recent years there has been much interest in the structures which underpin turbulent flows. These structures are often referred to as self-sustaining processes, coherent structures or vortex-wave interactions, and their common feature is that they apparently form at least 
part of the 'backbone' on which turbulent flows evolve. In fact it turns out that vortexwave interaction theory involving inviscid waves is simply the high Reynolds number description of the computationally-generated self-sustaining processes at finite Reynolds numbers; see for example Hall and Smith (1991), Hall and Sherwin (2010), Hall (2012a,b) for a discussion of vortex-wave interaction theory and Waleffe $(1997,2001,2003)$ for discussions of self-sustaining processes. Henceforth we refer to self-sustaining processes as SSP.

Although developed independently, the VWI theory of Hall and Smith (1989, 1990, 1991) can be viewed as a formal asymptotic approach to the type of meanflow-wave interaction proposed by Benney (1984), whilst the SSP is more closely related to the work of Nagata (1990) on finite amplitude perturbations to plane Couette flow. The common key process identified independently in VWI and SSP is the nonlinear coupling between an inviscid wave instability of a streak which reinforces the streak indirectly through its modification of the roll flow which then drives the streak. This three point coupling was revealed explicitly in the asymptotic theory of Hall and Smith (1991) whereas its recognition by Waleffe (1997) was a result of the interpretation of numerical results. The initial work of Hall and Smith (1991) simply derived the interaction equations in the context of wave interactions in compressible boundary layers but no solutions of the associated nonlinear eigenvalue problem were found until Hall and Sherwin (2010) applied the theory to plane Couette flow. The latter authors showed that the high Reynolds number assumption on which VWI is based leads to results which compare well with the full numerical SSP calculations of lower branch states by Wang, Gibson and Waleffe (2007). Application of SSP at high Reynolds number is made increasingly difficult because it must resolve vanishingly small internal layers where the flow changes rapidly. Thus, if one is interested in VWI/SSP states at Reynolds numbers greater than say $10^{5}$ in channel flows, only the VWI approach is as yet viable. Moreover, the remarkable agreement of the VWI results of Hall and Sherwin (2010) with the SSP results of Wang et al. (2007) down to Reynolds numbers quite close to the minimum one at which nonlinear states exist suggests it might sensibly be used more widely.

Most of the numerical work has been in relation to channel flow simulations but there exist many closely-related results for pipe flow; see for example Faisst and Eckhardt (2003), Wedin and Kerswell (2004), Kerswell and Tutty (2007), Viswanath (2009). For channel flows, Waleffe and co-workers, guided by the early ideas of Benney and colleagues, looked into the possibility that the Navier-Stokes equations might support wave systems occurring as instabilities of streamwise vortex flows but sufficiently large to drive the vortex flows; see for example Waleffe (1995, 1997, 1998, 2001, 2003), Wang et al. (2007). The approach used was to find fully nonlinear solutions of the Navier-Stokes equations using various fictitious forces to identify equilibrium states and then continue them when the forcing was switched off. Earlier Nagata (1990) had performed related calculations with the nonlinear flow initially driven by rotation and then connected to a finite amplitude state as the rotation decreased to zero. Much more detail of these structures was revealed subsequently in, for example, Clever and Busse (1997). Nagata's study was at the time open to what turned out to be unfounded criticism because of the lack of sufficient computational power to demonstrate the convergence of his results. Note also that the interpretation of the processes taking place in the equilibrium structures identified by Nagata was made clear by the work of Itano and Toh (2001) who conducted a computational investigation into the role of the equilibrium states in the Navier-Stokes dynamics.

The VWI work of Hall and Smith (1991), hereinafter referred to as HS1, built on related work by Hall and Smith $(1989,1990)$ and laid down a formal asymptotic framework for 
inviscid or viscous (Tollmien-Schlichting) waves interacting with roll-streak flows. Hall and Sherwin (2010), henceforth referred to as HS2, gave the first results for VWI applied to inviscid waves and showed that structure was the high Reynolds number version of what were referred to as lower branch states in the SSP community. This terminology is at odds with the usual one for linear stability theory applied to shear flows where lower branch modes correspond to viscous waves, i.e. Tollmien-Schlichting waves, whilst upper branch modes refer to waves with critical layers away from any boundaries and which are therefore predominantly inviscid in nature. We note that numerical simulations of fully turbulent flow require a minimum box size for sustained turbulence and this size is consistent with the upper limit of the streamwise wavenumber from the VWI approach of HS2.

It turns out that the lower branch states play a crucial role in delineating between initial perturbations to an undisturbed flow which remain laminar or become turbulent. Thus, if for example plane Couette flow is perturbed at time zero and the evolution of the flow calculated numerically, it turns out that a critical size exists for the initial amplitude of a given form of disturbance. Below this size the flow returns to the laminar flow state whilst above it the flow ultimately becomes turbulent. The nature of these edge states, and their complicated structure, has been investigated for plane Couette flow by Skufca, Yorke and Eckhardt (2006) and Schneider et al. (2008); see also Itano and Toh (2001) and Willis and Kerswell (2009) for other flow configurations.

More recently Duguet, Schlatter and Henningson (2009) and Schneider, Marinc and Eckhardt (2010a) independently carried out time-dependent integrations of the NavierStokes equations corresponding to initial streamwise and spanwise spatially localized perturbations to plane Couette flow. The simulations produced results similar to the edge state calculations of, for example, Skufca et al. (2006) but the edge state was now found to be also spatially localized whereas the latter authors found only edge states in small periodic cells. This result suggests that spatially-localized lower branch states might be found from either SSP or VWI and indeed Schneider et al. (2010b) found states spatially localized in the spanwise direction. Of interest here is the observation by Duguet et al. (2009) that turbulent patches formed during their calculations were elliptical in shape with the largest axis increasing with Reynolds number.

In a related problem of natural convection, Hall (2012a) has found that VWI states there have a tendency to localize as the spanwise wavenumber is decreased. However no VWI results for localization are available for plane Couette flow and analytical progress with natural convection was only made possible because VWI there persists to small amplitudes because of the inviscid instability of the unperturbed shear flow.

A notable feature of the results found by Duguet et al. (2009) was that the localized states have many similarities with turbulent spots found experimentally or for example numerically by Lundbladh and Johansson (1991). Thus, in the same way as NavierStokes simulations of turbulent plane Couette flow (Gibson et al. 2008), visit equilibrium states, it might be the case that the localized edge states found by Duguet et al. (2009) correspond to VWI states localized in the streamwise and spanwise directions and are in some sense attractors for flows exhibiting turbulent spots.

Since we concentrate on VWI theory in this paper, it is worth mentioning the key ideas that underpin the theory in the context of plane Couette flow. The basic idea is that across most of the channel the leading-order flow-field consists of a nonlinear $x$ independent roll-streak flow which evolves over a slow $O(R)$ timescale. The dependence on a slow scale results in the streak component of the flow dominating over the roll. This nearly-parallel flow is subjected to a small inviscid wave operating over $O(1)$ length and timescales. A critical layer is formed as a result and, provided the wave is of the 
appropriate amplitude, nonlinear wave interactions within this region lead to a jump in the roll flow across the critical layer. The roll-streak flow interacts back with the wave via the value of the streamwise shear stress of the streak at the critical layer, so that the system is fully interactive. More details of the process can be found in HS2 and in section 2 of this paper.

The main initial motivation for this work was to resolve a question left open in HS2. The question concerns the fate of inviscid waves participating in VWI in the limit of large streamwise wavelengths. The question is of some importance since the drag on the wall for plane Couette flow, say, has a minimum in the small wavenumber limit and so if turbulent flows are to be controlled then that regime might be of interest. In fact, as we shall see in section 2, the HS2 theory fails for plane Couette flow when the streamwise wavenumber is of size $R^{-1}$, and at this stage the critical layer associated with the wave thickens and fills the whole channel. We shall derive the appropriate interaction equations for this limit and solve them. In fact the interaction equations turn out to be the so-called 'boundary-region equations' or 'reduced Navier-Stokes equations' which are simply the Navier-Stokes equations without the perturbed streamwise pressure gradient and streamwise diffusion.

The interaction equations we derive in the long wave limit arise in a number of contexts and were derived by Hall (1988) in the context of Görtler vortices but with the Görtler number set equal to zero. The equations are also relevant to high Reynolds number flows in microchannels; see for example Fletcher (1991), Chen and Kuo (2004). In the context of vortex-wave interactions the equations were shown by Hall and Smith (1991) to govern the interaction of Tollmien-Schlichting waves and streaks. In the context of boundary-layer transition, the linearized version of the equations are relevant to the transient growth of streaks; see Luchini (2000), Higuera and Vega (2009), whilst the full nonlinear equations were solved by Zuccher, Tumin and Reshotko (2006) to compute optimal nonlinear streaks in boundary layers. The linearized version of the equations are also relevant to the receptivity of boundary layers to free stream turbulence; see for example Goldstein and Wundrow (1998), Goldstein and Sescu (2008). The key property of these equations is that they are parabolic in the flow direction and elliptic in the spanwise direction.

The long wave interaction equations we derive are solved numerically at different wavenumbers and we find that a streamwise localization process appears at sufficiently small streamwise wavenumber. Likewise, for a fixed downstream wavenumber, localization in the spanwise direction occurs as the spanwise wavenumber is decreased. The relationship of this structure with the numerical simulations of Duguet et al. (2009), Schneider et al. (2010a) will be discussed. At large values of the scaled streamwise wavenumber the results are consistent with the HS1 theory. In the latter limit the wave energy is confined to just the fundamental mode and the wave amplitude decreases in size compared to the roll flow thus recovering the asymptotic picture given in HS1.

The procedure adopted in the rest of this paper is as follows. In the following section we derive the VWI equations in the limit of small streamwise wavenumber: more precisely we investigate the case when the streamwise wavelength is comparable to the Reynolds number. In section 3 we discuss the numerical method used to solve the interaction equations, while in section 4 we present and discuss the results and draw some conclusions. 


\section{Formulation of the long wave interaction equations}

We consider viscous flow of kinematic viscosity $\nu^{*}$ in a channel occupying the region $-h<y^{*}<h$ in Cartesian coordinates $\left(x^{*}, y^{*}, z^{*}\right)$. The upper wall of the channel moves in the $x^{*}$ direction with constant velocity $U^{*}$, and the lower wall moves with velocity $-U^{*}$. If we non-dimensionalize our lengthscales by writing $\left(x^{*}, y^{*}, z^{*}\right)=h(\widehat{x}, \widehat{y}, \widehat{z})$ and employ the usual notation for the convective derivative, the Laplacian and the gradient operator, the required momentum and continuity equations take the form

$$
\begin{aligned}
\frac{D \widehat{\mathbf{u}}}{D \widehat{t}} & =-\nabla \widehat{p}+\frac{1}{R} \triangle \widehat{\mathbf{u}}, \\
\nabla \cdot \widehat{\mathbf{u}} & =0 .
\end{aligned}
$$

Here the dimensionless quantities $(\widehat{u}, \widehat{v}, \widehat{w})$ represent the fluid velocity and $\widehat{p}$ the pressure. The Reynolds number $R=U^{*} h^{*} / \nu^{*}$ and the time $t^{*}$ is written as $\left(h / U^{*}\right) \widehat{t}$. The appropriate no-slip boundary conditions at the channel walls are given by

$$
\widehat{\mathbf{u}}=( \pm 1,0,0) \text { on } \widehat{y}= \pm 1 .
$$

In the absence of any disturbance there exists a simple unidirectional basic solution $\left(u_{b}(\widehat{y}), 0,0\right)$ to $(2.1),(2.2)$, where

$$
u_{b}(\widehat{y})=\widehat{y}
$$

is the well-known plane Couette flow.

Now we will write down the VWI equations from HS2 appropriate to waves with streamwise and spanwise wavelengths comparable with the channel half-depth $h$. We first note that the Navier-Stokes equations (2.1) admit a form corresponding to a rollstreak solution of the type discussed in HS2 with

$$
\widehat{\mathbf{u}}=\left(u(\widehat{y}, \widehat{z}, \tau), R^{-1} v(\widehat{y}, \widehat{z}, \tau), R^{-1} w(\widehat{y}, \widehat{z}, \tau)\right), \widehat{p}=R^{-2} p(\widehat{y}, \widehat{z}, \tau),
$$

which satisfies the Navier-Stokes equations for all $R$. Here $\tau=R^{-1} \widehat{t}$ is a slow time scale and the flow is taken to be periodic in the spanwise direction with wavelength $2 \pi / \widehat{\beta}$. We now follow the analysis of HS1, HS2 and, assuming that the Reynolds number is large, we perturb the steady form of the roll-streak flow by writing

$$
\begin{aligned}
\widehat{\mathbf{u}} & =\left(u(\widehat{y}, \widehat{z}, \tau), R^{-1} v(\widehat{y}, \widehat{z}, \tau), R^{-1} w(\widehat{y}, \widehat{z}, \tau)\right)+\rho(\tau) R^{-7 / 6} \mathbf{U}(\widehat{y}, \widehat{z}, \tau) E+\text { c.c. }+\ldots, \\
\widehat{p} & =R^{-2} p(\widehat{y}, \widehat{z}, \tau)+\rho(\tau) R^{-7 / 6} P(\widehat{y}, \widehat{z}, \tau) E+\text { c.c. }+\ldots \\
E & \equiv \exp \left\{\mathrm{i} \widehat{\alpha}\left(\widehat{x}-R \int c(\tau) d \tau\right)\right\}
\end{aligned}
$$

so that the wave propagates in the $\widehat{x}$ direction with instantaneous speed $c$ and has wavenumber $\widehat{\alpha}$. The wave pressure $P$ satisfies a linear equation ((2.7) below) and is normalized at each value of $\tau$ such that $\iint|P|^{2} d \widehat{y} d \widehat{z}=1$. Here the integral is taken across the whole channel over one spanwise wavelength. The $O(1)$ quantity $\rho(\tau)$ represents the wave amplitude after normalization and is to be determined. Here we shall concentrate on waves with zero wavespeed but for completeness we shall initially show the form of the solution for travelling waves, which can exist in the present case or when the flow is driven by a pressure gradient. The size of the wave, $O\left(R^{-7 / 6}\right)$, is fixed as in HS1, HS2 in such a way that $O(1)$ jumps in the roll stresses are induced across the critical layer 
associated with the wave. The roll/streak field then satisfies

$$
\begin{aligned}
u_{\tau}+v u_{\widehat{y}}+w u_{\widehat{z}} & =u_{\widehat{y} \widehat{y}}+u_{\widehat{z} \widehat{z}}, \\
v_{\tau}+v v_{\widehat{y}}+w v_{\widehat{z}}+p_{\widehat{y}} & =v_{\widehat{y} \widehat{y}}+v_{\widehat{z} \widehat{z}}, \\
w_{\tau}+v w_{\widehat{y}}+w w_{\widehat{z}}+p_{\widehat{z}} & =w_{\widehat{y} \widehat{y}}+w_{\widehat{z} \widehat{z}}, \\
v_{\widehat{y}}+w_{\widehat{z}} & =0
\end{aligned}
$$

subject to

$$
u= \pm 1, v=w=0 \text { on } \widehat{y}= \pm 1 \text {. }
$$

Away from the critical layer the wave satisfies the linearized system

$$
\begin{aligned}
\mathrm{i} \widehat{\alpha}(u-c) U+V u_{\widehat{y}}+W u_{\widehat{z}} & =-\mathrm{i} \widehat{\alpha} P, \\
\mathrm{i} \widehat{\alpha}(u-c) V & =-P_{\widehat{y}}, \\
\mathrm{i} \widehat{\alpha}(u-c) W & =-P_{\widehat{z}}, \\
\mathrm{i} \widehat{\alpha} U+V_{\widehat{y}}+W_{\widehat{z}} & =0
\end{aligned}
$$

where $\mathbf{U}=(U, V, W)$. We see that at high Reynolds numbers the wave depends only on the streak velocity field and, following Hall and Horseman (1991), elimination of the wave velocity field leads to the wave pressure eigenvalue problem

$$
P_{\widehat{y} \widehat{y}}+P_{\widehat{z} \widehat{z}}-\widehat{\alpha}^{2} P-\frac{2 u_{\widehat{y}} P_{\widehat{y}}}{u-c}-\frac{2 u_{\widehat{z}} P_{\widehat{z}}}{u-c}=0, \text { with } P_{\widehat{y}}=0 \text { on } \widehat{y}= \pm 1 \text {. }
$$

In the spanwise direction we require that $P$ is periodic. Throughout the interaction the wave is taken to be instantaneously neutral, so that the wavespeed $c(\tau)$ remains real. The coupling between the wave and roll/streak flow is completed in the critical layer which occupies a region of width $R^{-1 / 3}$ around the location $\widehat{y}=f(\widehat{z}, \tau)$ where $u=c$. Here we will restrict our attention to the case when the wavespeed is zero and we will see that the special symmetries of the solution we construct lead to a flat critical layer with $f=0$. In the critical layer, we can deduce from (2.6) that the wave velocity components increase by $O\left(R^{1 / 3}\right)$, and the wave interacts with itself to drive the roll flow. When the roll flow inside the critical layer is matched to the outer flow, we see that the result of the wave driving the roll is to produce jumps in the stresses associated with the roll for the flow outside the critical layer. The velocity and pressure fields associated with the wave, roll and streak within the critical layer are found in exactly the same manner as given in HS1 and HS2 so we do not repeat the analysis here. The roll velocity components $(v, w)$ together with $u, u_{\widehat{y}}$ are continuous across the critical layer. However, following HS1, HS2, we find that the Reynolds stresses associated with the wave lead to the following jumps for the roll flow across the layer:

$$
\begin{aligned}
{\left[w_{\widehat{y}}\right]_{-}^{+} } & =\frac{\left[v_{\widehat{y}}\right]_{-}^{+}}{f_{\widehat{z}}}=J(\widehat{z}, \tau) \\
J & =\left(\frac{2}{3}\right)^{2 / 3} \Gamma\left(\frac{1}{3}\right) \frac{2 \pi \rho^{2}}{a^{5 / 3} \Theta^{5}}\left\{\left(-\frac{7 \Theta_{\widehat{z}}}{2 \Theta}-\frac{5 a_{\widehat{z}}}{3 a}\right)\left|P_{\widehat{z}}\right|^{2}+\frac{\partial}{\partial z}\left|P_{\widehat{z}}\right|^{2}\right\}, \\
{[p]_{-}^{+} } & =-\left(\frac{2}{3}\right)^{2 / 3} \Gamma\left(\frac{1}{3}\right) \frac{2 \pi \rho^{2}}{a^{5 / 3} \Theta^{5}} f_{\widehat{z} \widehat{z}}\left|P_{\widehat{z}}\right|^{2},
\end{aligned}
$$

where $\Theta=1+f_{\widehat{z}}^{2}, a=\mu \widehat{\alpha} / \Theta$ and $\mu$ is $\partial u / \partial \widehat{y}$ evaluated at $\widehat{y}=f(\widehat{z}, \tau)$. The \pm signs denote values immediately above and below the critical layer and the wave pressure $P$ in $(2.8)$ is to be evaluated on the critical layer. As explained in HS2, the easiest way to understand the origin of the jumps is by thinking of the flow in the critical 


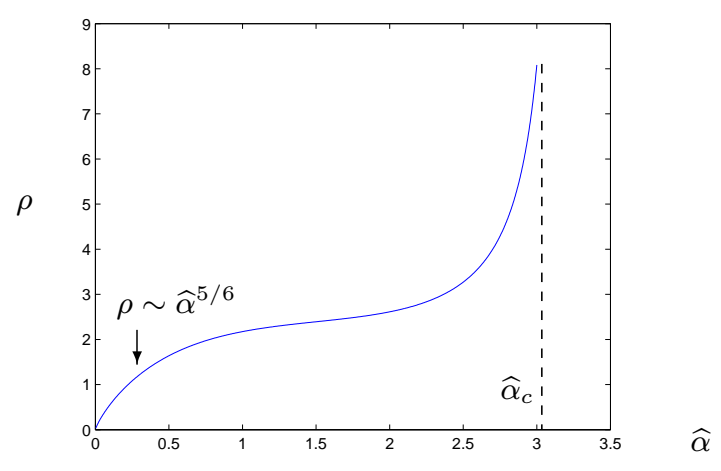

FIGURE 1. Wave amplitude $\rho$ versus wavenumber $\widehat{\alpha}$ from the VWI calculations of HS2, showing the critical wavenumber $\widehat{\alpha}_{c}$.

layer as a steady streaming problem. Thus in the critical layer the wavefield becomes relatively large and its interaction with itself generates Reynolds stresses which cause the roll velocity component parallel to the critical layer to have a jump in its normal derivative whilst the momentum equation normal to the layer produces a jump in the pressure. Thus the Reynolds stresses basically produce jumps in the roll stresses normal and parallel to the critical layer. Finally in this discussion of HS1, HS2 we note that the wave has a viscous layer at the walls of the same thickness as the critical layer. No jumps are produced there at leading order because the streamwise momentum equation shows that the leading-order wave pressure is zero in these layers. Full details of the numerical procedure needed to solve the nonlinear eigenvalue problem $\rho=\rho(\widehat{\alpha}, \widehat{\beta})$ can be found in HS2. For given values of the streamwise and spanwise wavenumbers $\widehat{\alpha}, \widehat{\beta}$, we can find $\rho$ and Figure 1 shows the typical behaviour with equilibrium states possible for $0<\widehat{\alpha}<\widehat{\alpha}_{c}$ for some critical wavenumber $\widehat{\alpha}_{c}$. It was also found in HS2 that the mean drag on the walls increases monotonically with $\widehat{\alpha}$ with the minimum drag therefore occurring at $\widehat{\alpha}=0$, according to this theory.

When $\widehat{\alpha}$ is sufficiently small however, the VWI scalings given in (2.4) break down. We can see this by first noting that for small values of wavenumber we can deduce from the jump conditions (2.8) and the wave equations (2.6) that

$$
\rho \sim \widehat{\alpha}^{5 / 6}, U \sim \widehat{\alpha}^{-2}, V \sim W \sim \widehat{\alpha}^{-1} \text { as } \widehat{\alpha} \rightarrow 0 .
$$

The distinguished scaling for $\widehat{\alpha}$ at which we enter a new regime can be most easily seen by comparing the contributions to the pressure from the roll and the wave in $(2.4 \mathrm{~b})$. These terms become comparable in size when $R^{-2} \sim \rho R^{-7 / 6}$, and hence upon use of (2.9) we see that a new stage is encountered when

$$
R^{-2} \sim \widehat{\alpha}^{5 / 6} R^{-7 / 6} \Rightarrow \widehat{\alpha} \sim R^{-1} .
$$

At this same stage the critical layer, formerly of thickness $O(\widehat{\alpha} R)^{-1 / 3}$ under the HS1 scalings, enlarges to fill the whole channel so that the wave is controlled by viscous effects everywhere. Note also that, away from the critical layer, the wave velocity components in the $\widehat{y}-\widehat{z}$ plane, which are of $O\left(R^{-7 / 6} \widehat{\alpha}^{-1 / 6}\right)$ from (2.4) and (2.9) as $\widehat{\alpha} \rightarrow 0$ in the HS1 regime, increase to size $O\left(R^{-1}\right)$, and are now the same magnitude as the roll velocity components. Similarly, an examination of (2.4a) shows that the streamwise component of the wave, which in the small wavenumber limit is of order $(\widehat{\alpha} R)^{-7 / 6}$ from $(2.9)$, becomes $O(1)$ and is therefore now comparable with the streak velocity. Thus when the wave 
has wavelength comparable with the Reynolds number it becomes viscous throughout the channel and the VWI mechanism of HS1 fails. In the new regime, the separation of scales between the wave velocity components and the underlying roll/streak flow disappears and the wave now forces the roll across the whole of the channel. In fact the new streamwise lengthscale is recognized as being the usual streamwise lengthscale over which Görtler vortices develop in curved flows; see Hall (1988). Hence the roll/streak flow which develops in the $\widehat{\alpha} \sim R^{-1}$ case can be thought of as a Görtler vortex driven by the stresses associated with the wave part of the flow, rather than by curvature. Note however that, for flows where Tollmien-Schlichting waves are possible, an intermediate interaction problem occurs much along the lines of the viscous VWI case described in HS1.

In view of the above discussion we now seek a solution of the Navier-Stokes equations in the form

$$
\begin{aligned}
& \widehat{\mathbf{u}}=\left(u(x, y, z, \tau), R^{-1} v(x, y, z, \tau), R^{-1} w(x, y, z, \tau)\right)\left(1+O\left(R^{-2}\right)\right), \\
& \widehat{p}=R^{-2} p(x, y, z, \tau)+O\left(R^{-4}\right), \\
& \tau=\widehat{t} / R, \quad x=\widehat{x} / R, \quad y=\widehat{y}, \quad z=\widehat{z}
\end{aligned}
$$

and substitution into (2.1) yields

$$
\begin{aligned}
\frac{D \mathbf{u}}{D \tau} & =-\left(0, p_{y}, p_{z}\right)+\left(\partial_{y y}^{2}+\partial_{z z}^{2}\right) \mathbf{u}, \\
\nabla \cdot \mathbf{u} & =0
\end{aligned}
$$

for $\mathbf{u}=(u, v, w)$, where

$$
\frac{D}{D \tau} \equiv \frac{\partial}{\partial \tau}+\mathbf{u} \cdot \nabla, \quad \nabla \equiv\left(\partial_{x}, \partial_{y}, \partial_{z}\right)
$$

Thus the leading order approximation to the VWI now reduces to the Navier-Stokes equations with the perturbed streamwise pressure gradient and streamwise diffusion negligible. However the crucial and indeed remarkable simplification in this limit is that the effective Reynolds number is now scaled to unity.

In addition to the vortex-Rayleigh wave application emphasized earlier in this paper, the equations studied here arise in many other instability problems involving flow at high Reynolds numbers. The equations themselves are easily generalized to a cylindrical geometry using the scalings (2.10), together with an $O(1)$ azimuthal variation. They take the form

$$
\begin{aligned}
u_{x}+v_{r}+v / r+w_{\theta} / r & =0, \\
u_{\tau}+u u_{x}+v u_{r}+w u_{\theta} / r & =K+\nabla^{2} u, \\
v_{\tau}+u v_{x}+v v_{r}+w v_{\theta} / r-w^{2} / r & =-p_{r}+\nabla^{2} v-v / r^{2}-2 w_{\theta} / r^{2}, \\
w_{\tau}+u w_{x}+v w_{r}+w w_{\theta} / r+v w / r & =-p_{\theta} / r+\nabla^{2} w-w / r^{2}+2 v_{\theta} / r^{2},
\end{aligned}
$$

where $\nabla^{2} \equiv \partial_{r r}^{2}+r^{-1} \partial_{r}+r^{-2} \partial_{\theta \theta}^{2}$, together with the no-slip condition on the boundaries, and regularity conditions along the pipe axis. The constant $K$ represents the axial pressure gradient associated with the basic flow. The above equations therefore control long wavelength lower branch equilibrium structures in Hagen-Poiseuille flow. The linearized version of (2.12) was shown to govern the neutral stability in pipes of general crosssection with aspect ratios of $O(1)$ by Smith (1979), but no numerical solution was given, although a multi-region asymptotic solution of (2.12) is believed to exist in the limit of large aspect ratio. The stability of developing flows is another area where (2.12) is relevant. For example, the linear stability of spatially-developing pipe and channel flows is 
governed by modes with an $O(R)$ wavelength, comparable to the lengthscale over which the basic flow is developing (Smith and Bodonyi 1980). The corresponding neutral modes in temporally-developing pipe flow exhibit the same behaviour (Walton 2002). A further situation where the linearized version of (2.12) is relevant is the stability of the axial flow in a concentric annulus, where the equations describe the travelling-wave instability close to the cut-off in radius ratio or sliding velocity (Gittler 1993, Walton 2004).

Returning now to the planar case, the major simplifications of (2.11) compared to the Navier-Stokes equations are that they do not contain the Reynolds number and the absence of a non-constant streamwise pressure gradient and streamwise diffusion renders them parabolic in $x$. Here we are interested in steady solutions of these equations which are periodic in $x$ and $z$. For large values of the scaled streamwise wavenumber $\alpha$ we also expect the solutions to match onto the small wavenumber $(\widehat{\alpha} \rightarrow 0)$ limit of the VWI problem of HS2. The major difficulty associated with these equations compared to the VWI case at $O(1)$ streamwise wavenumbers is that the roll/streak flow and wave cannot be separated and linked through jump conditions and indeed the wave can no longer be regarded as linear since all of its harmonics are now important.

\section{Numerical method}

Here we describe a numerical scheme to obtain travelling wave solutions of (2.11). The scheme is based on the tried and tested method outlined in Nagata (1990), in which the potential decomposition

$$
\begin{aligned}
\widehat{u} & =\overline{\widehat{u}}+\partial_{\widehat{x} \widehat{y}}^{2} \widehat{\phi}+\partial_{\widehat{z}} \widehat{\psi}, \\
\widehat{v} & =-\partial_{\widehat{x} \widehat{x}}^{2} \widehat{\phi}-\partial_{\widetilde{z} \widehat{z}}^{2} \widehat{\phi}, \\
\widehat{w} & =\overline{\widehat{w}}+\partial_{\widehat{y} \widehat{z}}^{2} \widehat{\phi}-\partial_{\widehat{x}} \widehat{\psi}
\end{aligned}
$$

is used for the full Navier-Stokes equations (2.1), and algebraic equations are calculated explicitly for their spectral coefficients and phase velocities. Here we seek the travelling wave solution which has wavenumber $\widehat{\alpha}$, phase velocity $\widehat{c}$ in the streamwise direction and wavenumber $\widehat{\beta}$, phase velocity $\widehat{c}_{z}$ in the spanwise direction. The operator

$$
\bar{*} \equiv \frac{\widehat{\alpha} \widehat{\beta}}{4 \pi^{2}} \int_{0}^{2 \pi / \widehat{\alpha}} \int_{0}^{2 \pi / \widehat{\beta}} * d \widehat{x} d \widehat{z}
$$

represents a spatial average over $\widehat{x}$ and $\widehat{z}$. We generate solutions to our problem $(2.11)$ by starting with known solutions of the full Navier-Stokes equations at finite Reynolds numbers, $\left(\widehat{\phi}, \widehat{\psi}, \widehat{u}, \widehat{w}, \widehat{c}, \widehat{c}_{z}\right)$, and using a homotopy to connect them to solutions of the required system. With that in mind we consider the modified equations

$$
\begin{aligned}
\frac{D \mathbf{u}}{D \tau} & =-\left(\epsilon p_{x}, p_{y}, p_{z}\right)+\triangle_{\epsilon} \mathbf{u}, \\
\nabla \cdot \mathbf{u} & =0, \\
\triangle_{\epsilon} & \equiv \epsilon \partial_{x x}^{2}+\partial_{y y}^{2}+\partial_{z z}^{2},
\end{aligned}
$$

with $\epsilon \equiv R^{-2}$. This augmented system is simply a rescaled form of the full NavierStokes equations (2.1), whilst upon setting $\epsilon=0$ we recover the system (2.11). To solve (3.2), the most natural definition of scaled potentials/mean-flows would be $(\widehat{\phi}, \widehat{\psi}, \overline{\widehat{u}}, \overline{\widehat{w}})=$ $\left(R^{-1} \phi, \psi, \bar{u}, R^{-1} \bar{w}\right)$, which does not change the form of (3.1) except for some factors of $\epsilon$ after the scaling. However, a minor change is necessary because in the limit $\epsilon \rightarrow 0$ one 
of the spanwise-averaged vorticity components,

$$
\Omega \equiv \frac{\widehat{\beta}}{2 \pi} \int_{0}^{2 \pi / \widehat{\beta}}\left\{\partial_{x} v-\partial_{y}(u-\bar{u})\right\} d \widehat{z}=-\epsilon \frac{\widehat{\beta}}{2 \pi} \int_{0}^{2 \pi / \widehat{\beta}}\left(\partial_{x x x}^{3}+\partial_{x y y}^{3}\right) \phi d \widehat{z},
$$

vanishes identically and the Jacobian matrix appearing in our Newton procedure will therefore be singular. In order to account for this, we use a modified scaling for the spanwise-averaged poloidal potential and write

$$
\begin{aligned}
u & =\bar{u}+\epsilon \partial_{x y}^{2} \phi+\partial_{z} \psi+\partial_{y} \varphi \\
v & =-\epsilon \partial_{x x}^{2} \phi-\partial_{z z}^{2} \phi-\partial_{x} \varphi \\
w & =\bar{w}+\partial_{y z}^{2} \phi-\partial_{x} \psi
\end{aligned}
$$

The relationship between the solutions of the Navier-Stokes equations (2.1) and the augmented system (3.2) is

$$
\left(\widehat{\phi}-\widehat{\varphi}, \partial_{\widehat{x}} \widehat{\varphi}, \widehat{\psi}, \overline{\widehat{u}}, \overline{\widehat{w}}, \widehat{c}, \widehat{c}_{z}\right)=\left(R^{-1} \phi, \varphi, \psi, \bar{u}, R^{-1} \bar{w}, c, R^{-1} c_{z}\right)
$$

where $\widehat{\varphi}=\frac{\widehat{\beta}}{2 \pi} \int_{0}^{2 \pi / \widehat{\beta}} \widehat{\phi} d \widehat{z}$. It is easy to confirm that $\Omega=-\left(\partial_{x x}^{2}+\partial_{y y}^{2}\right) \varphi$ does not vanish at $\epsilon=0$ and so the problem of the singular Jacobian has been overcome.

The governing equations in terms of potentials are found by considering the operations $\mathbf{e}_{y} \cdot \nabla_{\epsilon} \times \nabla \times(3.2 a), \mathbf{e}_{y} \cdot \nabla_{\epsilon} \times(3.2 a), \mathbf{e}_{x} \cdot \overline{(3.2 a)}$ and $\mathbf{e}_{z} \cdot \overline{(3.2 a)}$ and take the form

$$
\begin{gathered}
\left(c \partial_{x}+c_{z} \partial_{z}+\triangle_{\epsilon}\right) \triangle_{\epsilon}\left(\triangle_{\epsilon}^{2 D} \phi+\partial_{x} \varphi\right)+\triangle_{\epsilon}((\mathbf{u} \cdot \nabla) v)-\partial_{y}(\nabla \cdot((\mathbf{u} \cdot \nabla) \mathbf{u}))=0 \\
\left(c \partial_{x}+c_{z} \partial_{z}+\triangle_{\epsilon}\right) \triangle_{\epsilon}^{2 D} \psi+\epsilon \partial_{x}((\mathbf{u} \cdot \nabla) w)-\partial_{z}((\mathbf{u} \cdot \nabla) u)=0 \\
\partial_{y y}^{2} \bar{u}-\partial_{y} \overline{v u}=0 \\
\partial_{y y}^{2} \bar{w}-\partial_{y} \overline{v w}=0 \\
\phi=\partial_{y} \phi=\varphi=\partial_{y} \varphi=\psi=\bar{w}=0, \bar{u}= \pm 1 \text { on } y= \pm 1
\end{gathered}
$$

where $\nabla_{\epsilon} \equiv\left(\epsilon \partial_{x}, \partial_{y}, \partial_{z}\right)$ and $\triangle_{\epsilon}^{2 D} \equiv \epsilon \partial_{x x}^{2}+\partial_{z z}^{2}$. Here a Galilean transformation has been applied in the $x$ and $z$ directions so that the problem becomes time independent. For numerical purposes, the potentials and mean-flows are approximated by the truncated spectral expansions

$$
\begin{aligned}
& \phi=\sum_{\substack{m=-M \\
n \neq 0}}^{M} \sum_{\substack{n=-N \\
l=0}}^{N} X_{l m n}^{(1)} \Phi_{l}(y) e^{\mathrm{i} m \alpha x} e^{\mathrm{i} n \beta z}, \\
& \varphi=\sum_{\substack{m=-M \\
m \neq 0}}^{M} \sum_{\substack{l=0 \\
(m, n) \neq(0,0)}}^{L} \alpha^{-1} X_{l m 0}^{(1)} \Phi_{l}(y) e^{\mathrm{i} m \alpha x}, \\
& \psi=\sum_{\substack{m=-M \\
(m=-N}}^{N} \sum_{l=0}^{L} \alpha^{-1} X_{l m n}^{(2)} \Psi_{l}(y) e^{\mathrm{i} m \alpha x} e^{\mathrm{i} n \beta z}, \\
& \bar{u}=y+\sum_{l=0}^{L} X_{l 00}^{(1)} \Psi_{l}(y), \\
& \bar{w}=\sum_{l=0}^{L} X_{l 00}^{(2)} \Psi_{l}(y),
\end{aligned}
$$

with $\alpha, \beta$ the scaled wavenumbers in the $x, z$ directions and where $\Phi_{l}(y) \equiv\left(1-y^{2}\right)^{2} T_{l}(y)$ 
and $\Psi_{l}(y) \equiv\left(1-y^{2}\right) T_{l}(y)$ are modified $l$ th Chebyshev polynomials of the first kind. Note also that because of the rescaling in $x$ given in (2.10), the wavelength in the streamwise direction is $2 \pi / \alpha=2 \pi R / \widehat{\alpha}$, whereas the spanwise scale is unchanged, i.e. we have

$$
\widehat{\alpha}=\alpha R, \widehat{\beta}=\beta .
$$

Numerically we find that equilibrium solutions typically have $\alpha \sim O\left(10^{2}\right)$ : since $O(\phi) \sim$ $O(\alpha \varphi) \sim O(\alpha \psi)$ as $\epsilon \rightarrow 0$, we introduce the scaling factor $\alpha^{-1}$ in (3.6b) and (3.6c) in order to improve the condition number of the Jacobian matrix. If we now make a Galerkin projection $\left\langle * e^{-\mathrm{i} m_{0} \alpha x} e^{-\mathrm{i} n_{0} \beta z} \Phi_{l_{0}}(y) \varrho(y)\right\rangle$ for $(3.5 a)$ and $\left\langle * e^{-\mathrm{i} m_{0} \alpha x} e^{-\mathrm{i} n_{0} \beta z} \Psi_{l_{0}}(y) \varrho(y)\right\rangle$ for $(3.5 b),(3.5 c)$ and $(3.5 d)$ with volumetric average operator $\langle *\rangle \equiv(1 / 2) \int_{-1}^{1} \bar{*} d y$ and weight function $\varrho(y) \equiv 2 /\left(\pi \sqrt{1-y^{2}}\right)$, we obtain a set of algebraic equations $F_{i}=D_{i j} X_{j}+$ $H_{i j k} X_{j} X_{k}=0$ as found in Nagata (1990).

Given a solution of (2.1) we can generate values of the solution vector $X_{j}$ by using the relation (3.4). Therefore, in principle, we can obtain a finite-amplitude solution of (3.2) for $\epsilon=0$ by a homotopy method starting from some known solution. The solution is continued by Newton's method, i.e. $X_{j}$ is updated as $X_{j}^{I+1}=X_{j}^{I}+q \delta X_{j}^{I}$ at each Newton iteration by solving the linear problem

$$
G_{i j}^{I} \delta X_{j}^{I}=-F_{i}^{I}
$$

by LAPACK package DGESVX. Here $G_{i j}^{I}=D_{i j}+2 H_{i j k} X_{k}^{I}$, the Jacobian matrix, and $F_{i}^{I}=D_{i j} X_{j}^{I}+H_{i j k} X_{j}^{I} X_{k}^{I}$ are computed by using double precision FORTRAN code. The coefficient $q$ is usually chosen as 1 , but if the prediction is overestimated, $q$ is chosen as some smaller value. This scheme is iterated until the convergence criteria

$$
r=\max _{j} r_{j}<10^{-5}
$$

is attained, where

$$
r_{j}=\left\{\begin{array}{cl}
\left|\frac{X_{j}^{I+1}-X_{j}^{I}}{X_{j}^{I}}\right| & \text { if } \quad\left|X_{j}^{I+1}\right| \text { and }\left|X_{j}^{I}\right|>10^{-10} \\
0 & \text { otherwise }
\end{array}\right.
$$

is satisfied.

The lower branch mirror symmetric steady solution of (2.1) found by Gibson et al. (2009) and Itano and Generalis (2009) is used as input to the scaled system (3.5) to provide the starting point of the homotopy. It is known that this type of solution is stationary, i.e. $c=c_{z}=0$, and has the following symmetries:

$$
\begin{aligned}
& {[u, v, w](x, y, z)=[u, v,-w](x+\pi / \alpha, y,-z)} \\
& {[u, v, w](x, y, z)=[-u,-v, w](-x,-y, z+\pi / \beta)} \\
& {[u, v, w](x, y, z)=[u, v, w](x+\pi / \alpha, y, z+\pi / \beta)}
\end{aligned}
$$

By using these symmetries, all the redundant unknowns are removed from the system to reduce the size of the calculation. For further cost reduction, we use an elliptic cut-off method, i.e. the coefficients and equations which satisfy $\left(\frac{l}{L+1}\right)^{2}+\left(\frac{m}{M+1}\right)^{2}+\left(\frac{n}{N+1}\right)^{2}>$ 1 are omitted. We choose the solution at $R=700$ with optimum wavenumber pair $(\widehat{\alpha}, \widehat{\beta})=(0.75,1.37)$ as the starting point of the homotopy of the augmented system $(3.2)$ for $(\alpha, \beta)=(525,1.37)$. Decreasing $\epsilon$ from $700^{-2} \simeq 2.04 \times 10^{-6}$, the branch successfully crosses $\epsilon=0$ using a relatively low resolution. Then at $\epsilon=0$, spectral accuracy is refined. 
We set $\epsilon=0$ henceforth. We consider the mean shear stress on the wall

$$
\Delta=\left.\partial_{y} \bar{u}\right|_{y=1}=1-2 \sum_{l=0}^{L} X_{l 00}^{(1)}
$$

and the $L_{2}$-norm of the spectral coefficients

$$
\left\|X_{j}\right\|_{2}=\sqrt{\sum_{j=1}^{\operatorname{dim}}\left|X_{j}\right|^{2}}
$$

as measures of the nonlinear solutions.

The result of resolution tests for $\alpha=500$ are shown in Table 1 . Since our convergence criteria does not involve the value of $\left\|F_{j}\right\|_{2}$, we show this together with its linear part $\left\|D_{j k} X_{k}\right\|_{2}$ in the table. Readers who are concerned about the signal-to-noise-floor ratio of the solution can easily confirm that our convergence criteria assure a reasonably small normalized residual $\left\|F_{j}\right\|_{2} /\left\|D_{j k} X_{k}\right\|_{2}$. The results presented in Table 1 suggest that an increase in resolution cannot remove fluctuation in the third digit in terms of $\Delta$ and in the fourth digit in terms of $\left\|X_{j}\right\|$. In order to convince the reader about the accuracy of our calculations we make a couple of observations. The first concern is that of the ill-conditioned Jacobian matrix associated with the Newton iteration. It is found that the typical condition number of this problem is around $10^{13} \sim 10^{15}$. However, the use of quadruple precision and/or stricter convergence criteria for the Newton iterations did not alter the leading digits of the results. The other concern is the convergence property of the spectral expansion, because of the lack of dissipation in the $x$ direction. To study the spectral convergence against the truncation level $L, M, N$, the following partial sums are introduced:

$$
\begin{aligned}
S_{l} & =\sqrt{\sum_{m=-M}^{M} \sum_{n=-N}^{N}\left|X_{l m n}^{(1)}\right|^{2}+\left|X_{l m n}^{(2)}\right|^{2}}, \\
S_{m} & =\sqrt{\sum_{l=0}^{L} \sum_{n=-N}^{N}\left|X_{l m n}^{(1)}\right|^{2}+\left|X_{l m n}^{(2)}\right|^{2}}, \\
S_{n} & =\sqrt{\sum_{l=0}^{L} \sum_{m=-M}^{M}\left|X_{l m n}^{(1)}\right|^{2}+\left|X_{l m n}^{(2)}\right|^{2} .}
\end{aligned}
$$

Note that $S_{m}=S_{-m}$ and $S_{n}=S_{-n}$. We can see from figure 2 that as the truncation level is increased, the magnitude of coefficients is almost monotonically decreasing whereas the leading order terms are unchanged except for a tiny variation. Hence we can conclude that the current computation is reliable within the realm of the indicated error levels. The truncation level $(L, M, N)=(70,16,30)$ is considered to be reliable for $\alpha=500$. It is found however that we need more harmonics in the $x$ direction as $\alpha$ is decreased. We exhibit another resolution test at $\alpha=100$ (Table 2) and set $(L, M, N)=(70,34,28)$ for $\alpha<100$. We can see that the quantity $\left\|F_{j}\right\|_{2} /\left\|D_{j k} X_{k}\right\|_{2}$ also stays sufficiently small in this case. However, as $\alpha$ is decreased and $M$ increased, the condition number becomes worse and some coefficients never achieve the convergence criteria. For this reason, at small $\alpha$, we have to switch to convergence criteria expressed in terms of $\left\|F_{j}\right\|_{2} /\left\|D_{j k} X_{k}\right\|_{2}$.

The use of the perturbed Newton's method introduced recently by Peris et al. (2011) helps to achieve a decrease in $\left\|F_{j}\right\|_{2}$. In this method, the correction $\delta X_{j}^{I}$ for $I \neq 0$ is solved by using the perturbed Jacobian matrix $\tilde{G}_{i j}^{I}=D_{i j}+2 H_{i j k} \tilde{X}_{k}^{I}$ instead of the 


$\begin{array}{cccccc}(L, M, N) & \operatorname{dim} & \Delta-1 & \left\|X_{j}\right\|_{2} & \left\|D_{j k} X_{k}\right\|_{2} & \left\|F_{j}\right\|_{2} \\ (60,30,34) & 35066 & 4.145 \times 10^{-2} & 12.9422 & 1.24 \times 10^{3} & 8.08 \times 10^{-12} \\ (70,16,30) & 19766 & 4.168 \times 10^{-2} & 12.9699 & 1.26 \times 10^{3} & 1.04 \times 10^{-11} \\ (70,18,32) & 23495 & 4.116 \times 10^{-2} & 12.9072 & 1.21 \times 10^{3} & 1.77 \times 10^{-8} \\ (70,18,34) & 24955 & 4.113 \times 10^{-2} & 12.9029 & 1.21 \times 10^{3} & 8.81 \times 10^{-12} \\ (85,16,30) & 23904 & 4.135 \times 10^{-2} & 12.9292 & 1.23 \times 10^{3} & 8.77 \times 10^{-11} \\ (85,18,32) & 28402 & 4.131 \times 10^{-2} & 12.9243 & 1.22 \times 10^{3} & 1.60 \times 10^{-11} \\ (85,18,34) & 30148 & 4.128 \times 10^{-2} & 12.9217 & 1.22 \times 10^{3} & 2.52 \times 10^{-11} \\ (95,16,30) & 26653 & 4.125 \times 10^{-2} & 12.9179 & 1.22 \times 10^{3} & 3.19 \times 10^{-8} \\ (95,18,32) & 31687 & 4.116 \times 10^{-2} & 12.9585 & 1.25 \times 10^{3} & 5.23 \times 10^{-9} \\ (95,18,34) & 33613 & 4.136 \times 10^{-2} & 12.9306 & 1.23 \times 10^{3} & 2.86 \times 10^{-9}\end{array}$

TABLE 1 . The convergence of the longwave high $R$ limit solution. $(\alpha, \beta)=(500,1.37)$.

$\begin{array}{cccccc}(L, M, N) & \operatorname{dim} & \Delta-1 & \left\|X_{j}\right\|_{2} & \left\|D_{j k} X_{k}\right\|_{2} & \left\|F_{j}\right\|_{2} \\ (95,18,34) & 33613 & 1.396 \times 10^{-2} & 4.6817 & 1.73 \times 10^{3} & 7.04 \times 10^{-9} \\ (80,24,34) & 37417 & 1.108 \times 10^{-2} & 4.2844 & 1.08 \times 10^{3} & 1.67 \times 10^{-8} \\ (70,30,34) & 40754 & 1.128 \times 10^{-2} & 4.3108 & 1.12 \times 10^{3} & 4.00 \times 10^{-9} \\ (70,34,28) & 38125 & 1.113 \times 10^{-2} & 4.3090 & 1.12 \times 10^{3} & 1.24 \times 10^{-11}\end{array}$

TABLE 2. The convergence of the longwave high $R$ limit solution. $(\alpha, \beta)=(100,1.37)$.

exact Jacobian matrix $G_{i j}^{I}$, where $\tilde{X}_{j}^{I}$ is the perturbed solution obtained by $G_{i j}^{0}\left(\tilde{X}_{j}^{I}-\right.$ $\left.X_{j}^{I}\right)=-F_{j}^{I}$. Obviously the success of this method depends on the proximity of the initial condition to the desired solution. We perturbed $X_{j}^{0}$ by an $O\left(10^{-7}\right)$ random disturbance during the tracing of the bifurcation curves, and confirmed that the fluctuation during the iterations did not affect the present accuracy of $\Delta$. The quantity $\left\|F_{j}\right\|_{2} /\left\|D_{j k} X_{k}\right\|_{2}$ is $O\left(10^{-6}\right)$ at worst for the results we present in the next section.

\section{Results and discussion}

Once the results for $\beta=1.37$ were found then $\alpha$ was fixed and $\beta$ varied until $\beta=1$. For this new value of $\beta$ we then varied $\alpha$. We then varied $\beta$ with $\alpha=100$ until $\beta=0.5$ but it is difficult to vary $\alpha$ with this spanwise wave number because (i) we need to increase $N$ to 40 and the computation then takes an inordinate amount of time; (ii) the ill-conditioned nature of the Newton method described in the previous section becomes worse and it becomes difficult to obtain reliable solutions for small $\alpha$. In view of this difficulty we show only three results for this value of $\beta$. The results of these calculations for different $\beta$ are shown in Figure 3 where the mean wall shear $\Delta$ is plotted as a function of the scaled wavenumber $\alpha$. For large values of $\alpha$ the shear approaches a constant value greater than the basic flow value of unity consistent with the prediction of the VWI approach described in HS2. An examination of the velocity field showed that, as in HS2, only the fundamental mode in the streamwise direction was significant. We conclude then that the minimum drag for lower branch states is predicted by the zero streamwise wavenumber limit of HS2. For smaller values of the scaled wavenumber $\alpha$ we see that the shear increases rapidly and the results suggest it becomes infinite at a finite value of $\alpha=\alpha_{c} \sim 70$ for the case $\beta=1.37$. This suggests that as well as the minimum box 

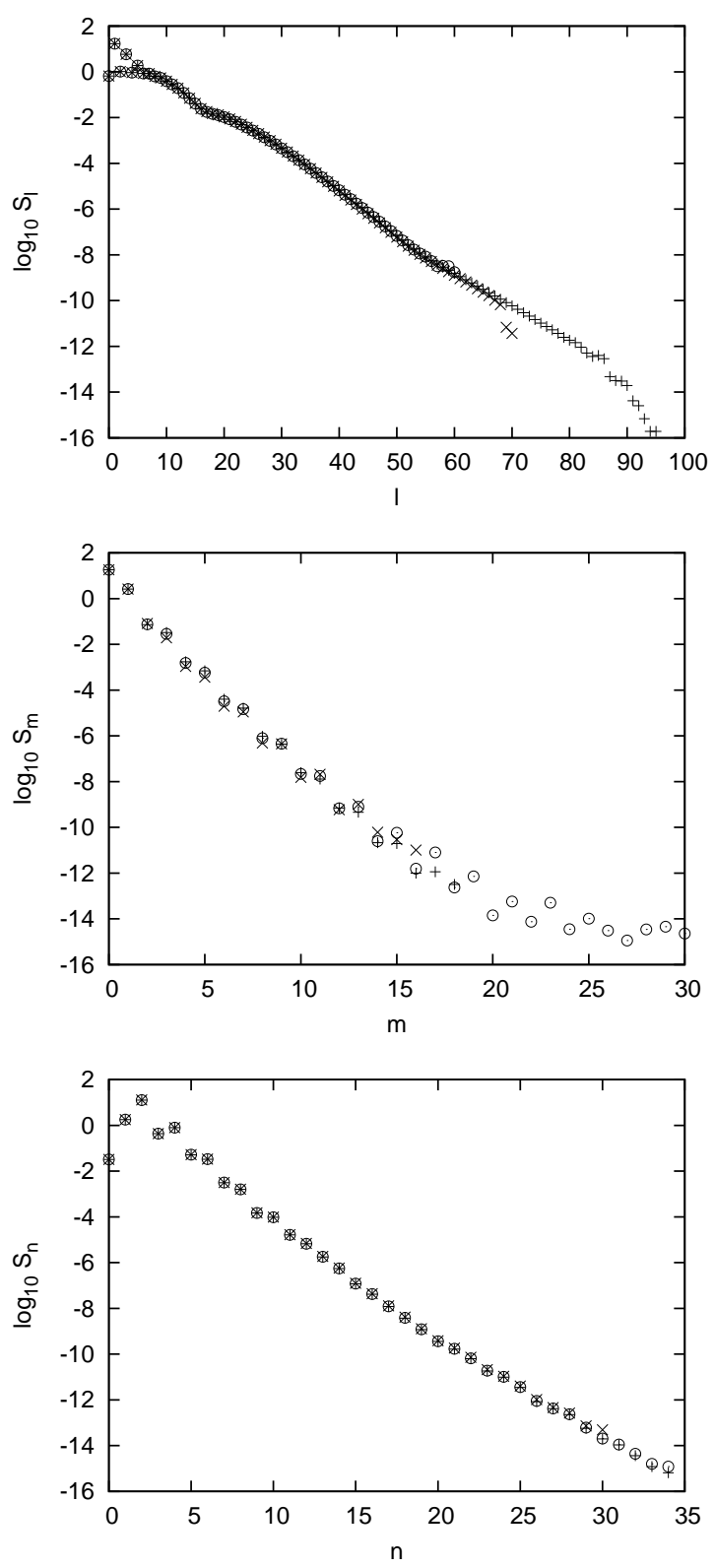

Figure 2. The partial sums of the spectral coefficients. $(\alpha, \beta)=(500,1.37)$. The spectral convergence: $\times,+$ and $\circ$ are calculated by $(L, M, N)=(70,16,30),(L, M, N)=(95,18,34)$ and $(L, M, N)=(60,30,34)$ respectively.

size for equilibrium states already predicted by HS2, there is also a critical size given by $2 \pi R / \alpha_{c}$ at which a significant change in the nature of the equilibrium states occurs; the nature of that change will become clear in the following discussion.

We expect the result described above for $\beta=1.37$ to be true for all values of the spanwise wavenumber for which the HS2 theory applies. We certainly expect that the two curves shown are representative and that for each $\beta$ the shear approaches the HS2 value at large $\alpha$ and blows up at some value of $\alpha$ in the region $50-70$. In Figure 3 we 


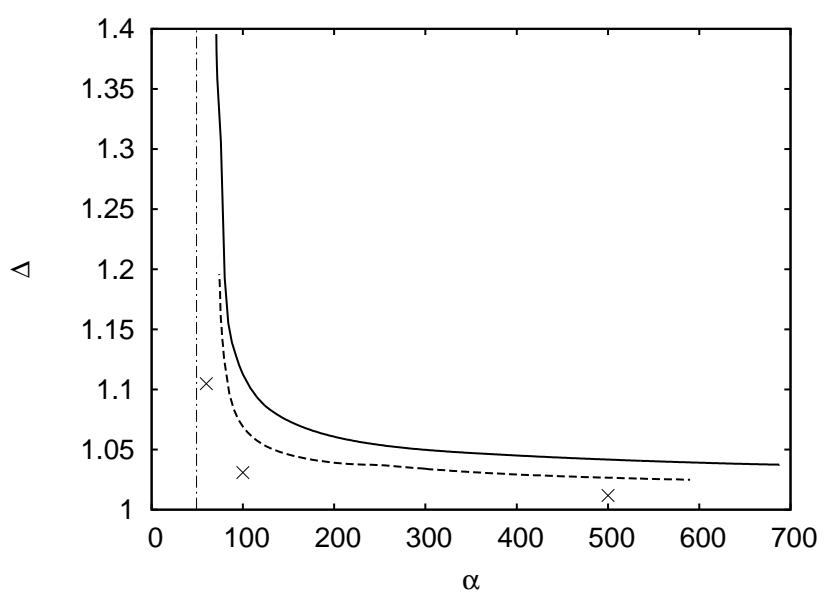

FIgURE 3 . The mean wall shear $\Delta$. The solid and dashed curves are $\beta=1.37$ and 1 respectively. The vertical dash-dotted line is the $2 \mathrm{D}$ energy threshold. The crosses represent the results for $\beta=0.5$, where resolution is chosen as $(L, M, N)=(70,40,40)$ for $\alpha=60$ and 100 whereas $(L, M, N)=(75,24,50)$ for $\alpha=500$.

also show as a vertical line the value of $\alpha$ below which energy theory predicts stability (see the appendix for more details); our solutions can therefore not cross this line.

Figures 4, 5, 6 show contour plots of the velocity components for the cases $\alpha=500,71$ with $\beta=1.37$. Figure $6 \mathrm{~b}$ offers a clue to the change in the nature of the equilibrium state as the limiting wavenumber $\alpha_{c}$ is approached. We refer to the fact that there appears to be a localization developing in the streamwise direction of the disturbance structure. This suggests that the energy of the disturbance is moving into the higher streamwise harmonics as the limiting solution is approached. In order to demonstrate that this is indeed the case we show in Figure 7 plots of the energy in the different modes as $\alpha$ is decreased. For the highest value of $\alpha$ we see that the energy is concentrated in the fundamental as predicted by the HS2 theory. At the lower value of the wavenumber we now see that the energy is spreading to the higher harmonics: this accounts for the increasing localization of the flow in the streamwise direction.

In order to see the localization more clearly, we have in Figure 8 plotted contours of the streamwise velocity component in the mid-plane $y=0$ for $\alpha=500,300,100$ and 71 with $\beta=1.37$. Note that the unperturbed flow is zero here so the figure shows how the equilibrium $x$-velocity component develops a localization in the streamwise direction. Notice also that in our discussion it should be remembered that the streamwise scale should be multiplied by $R$ so that Figure 8 represents elongated structures which develop small regions where the activity becomes concentrated as $\alpha$ decreases. Figure 9 shows similar results for the case $\alpha=100$, but now for two smaller values of spanwise wavenumber $\beta=1.0,0.5$. We now observe that the localization is also taking place in the spanwise direction as $\beta$ is decreased. This localization can perhaps be even more clearly seen in Figure 10 where, for a range of values of $\alpha$ and $\beta$, we show the regions where the total streamwise vorticity is greater than 80 per cent of its maximum absolute value.

The short scale structures have similar shapes to turbulent spots that have been observed experimentally in plane Couette flow; see for example Tillmark and Alfredsson (1992), Tillmark (1995). Turbulent spots have been simulated computationally by, for example, Lundbladh and Johansson (1991). Thus it is natural to expect some connection between these spots and our solutions. 
(a)

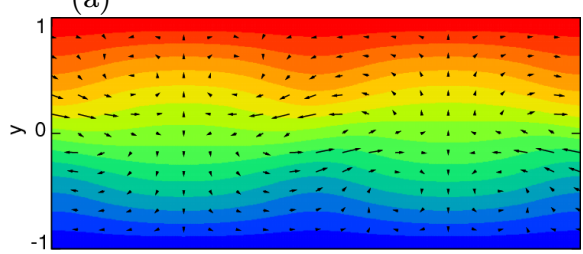

(c)

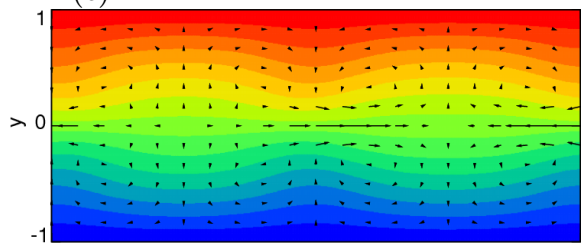

(e)

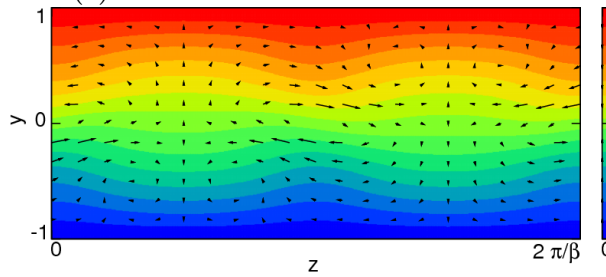

(b)

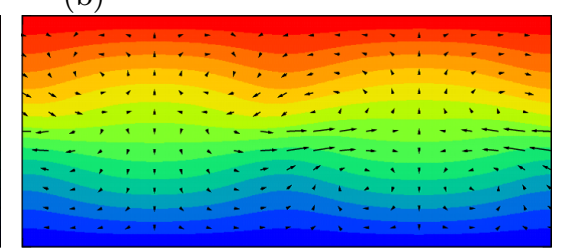

(d)

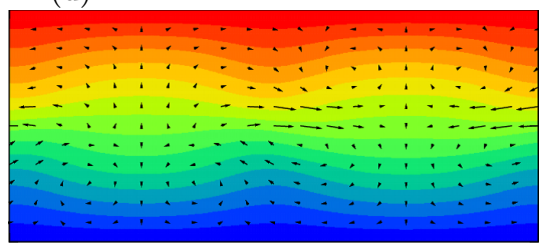

(f)

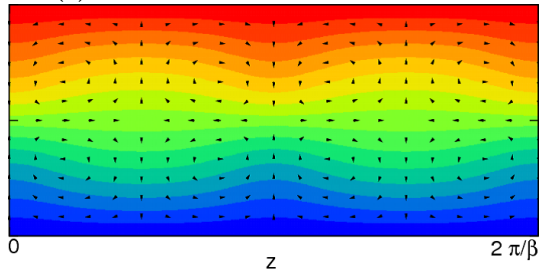

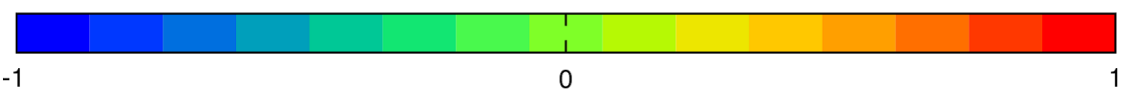

FIgURE 4. The velocity field $\mathbf{u}$ for $(\alpha, \beta)=(500,1.37)$. The colours represent the streamwise component $u$ whereas the arrows are cross-sectional components $v, w$. (a), (b), (c), (d) and (e) correspond to streamwise cross-sections at $x=(n / 8)(2 \pi / \alpha)$ with $n=0,1,2,3$ and 4 respectively. (f) is the streamwise-averaged field.

Further evidence for that possibility can be found in recent papers by Duguet et al. (2009) and Schneider et al. (2010a). The latter authors conducted direct numerical simulations of plane Couette flow restricting flow dynamics in some symmetric subspace. The initial state was taken to be localized in the spanwise and streamwise directions and by carefully altering the initial states the authors were able to generate 'edge states' which have similarities with turbulent spots. In the same way as the edge state calculations of Schneider et al. (2008), who did not impose symmetry, generated states similar to the lower branch state found by Wang et al. (2007) numerically and by HS2 asymptotically, we might conclude that the localized states found in this paper are related to the states tracked by Duguet et al. (2009) and Schneider et al. (2010a). We note however that the latter two publications concentrated on initial perturbations localized in both the streamwise and spanwise directions whereas our results for $\beta=1.37$ and 1.0 correspond to states which are just localized in the streamwise direction, but certainly the result shown for $\beta=0.5$ demonstrates the localization beginning to occur in the spanwise direction as $\beta$ decreases.

For plane Couette flow with downstream wavenumbers of $O(1)$ we know that the lower branch states of HS2 or equivalently those of Wang et al. (2007) are visited in unsteady Navier-Stokes simulations of turbulence (see Gibson et al. 2008). The nature of the localized solutions we have uncovered suggests that the interaction problem we have formulated has streamwise-localized states which might well be weakly unstable saddle-like points for trajectories of the unsteady Navier-Stokes equations. What we are 
(a)

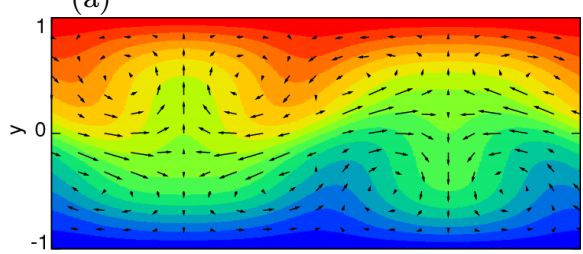

(c)

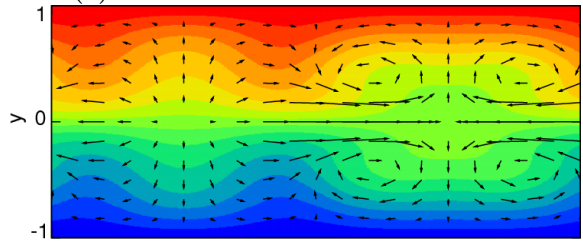

(e)

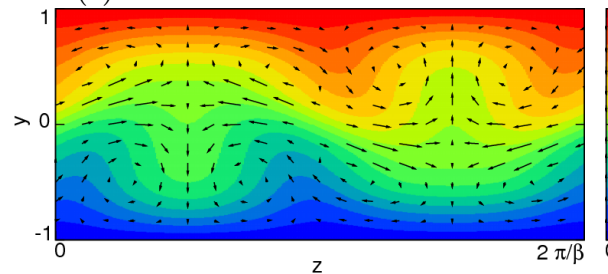

(b)

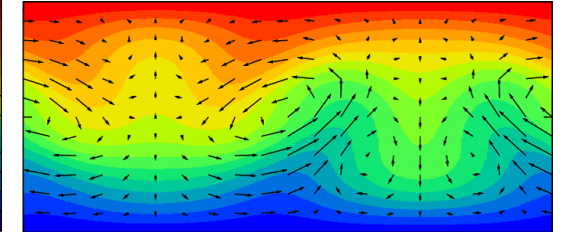

(d)

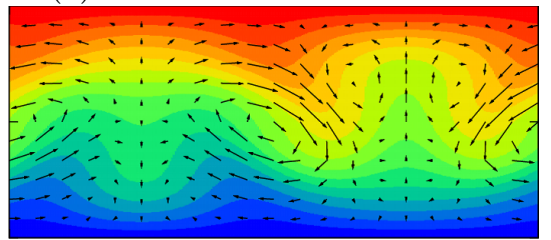

(f)

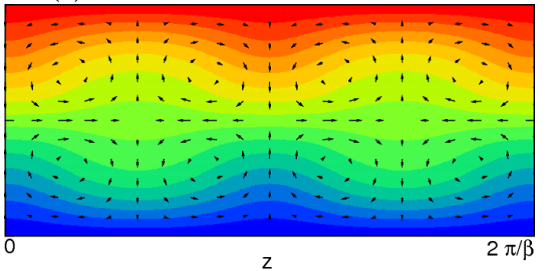

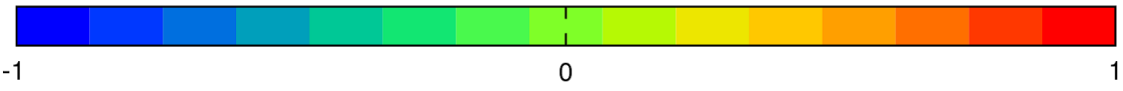

FIgURE 5. The velocity field $\mathbf{u}$ for $(\alpha, \beta)=(71,1.37)$. The colours represent the streamwise component $u$ whereas the arrows are cross-sectional components $v, w$. (a), (b), (c), (d) and (e) correspond to streamwise cross-sections at $x=(n / 8)(2 \pi / \alpha)$ with $n=0,1,2,3$ and 4 respectively. (f) is the streamwise-averaged field.

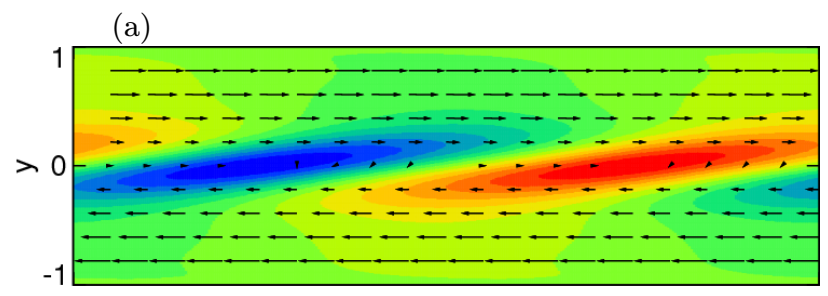

(b)

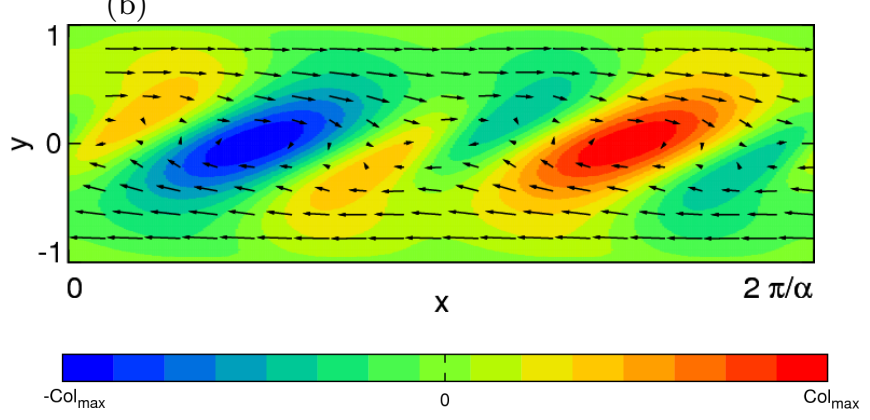

Figure 6. The velocity field $\mathbf{u}$ at $z=0$. The colours represent the spanwise component $w$ whereas the arrows are cross-sectional components $u, v / \alpha .(\mathrm{a}):(\alpha, \beta)=(500,1.37)$, $\mathrm{Col}_{\max }=18.9$. (b): $(\alpha, \beta)=(71,1.37), \mathrm{Col}_{\max }=36.7$. 


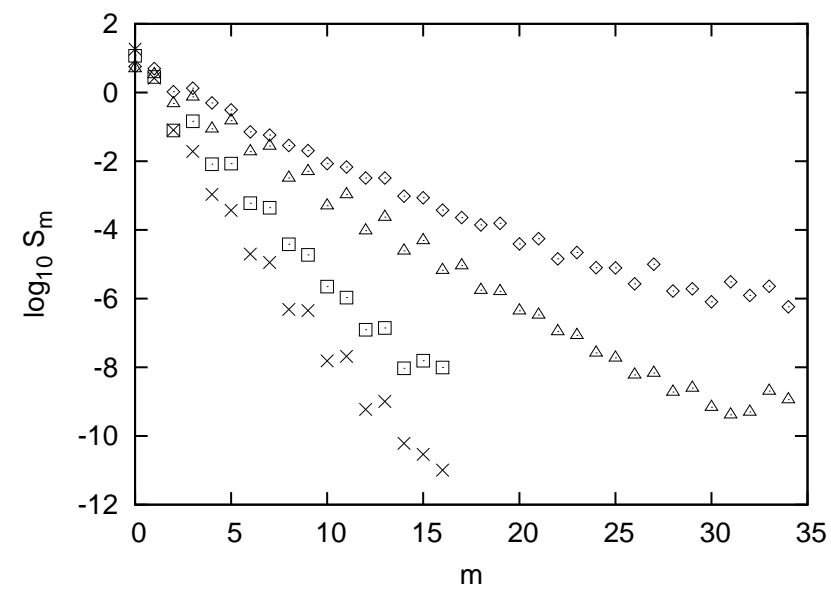

FiguRE 7. The partial sums of the spectral coefficients. $(\alpha, \beta)=(500,1.37)$. The spectral convergence: $\times, \square, \triangle$ and $\diamond$ are $\alpha=500,300,100$ and $71 .(L, M, N)=(70,16,30)$ is used for $\alpha=500$ and 300 whereas $(L, M, N)=(70,34,28)$ is used for $\alpha=100$ and 71 .

(a)

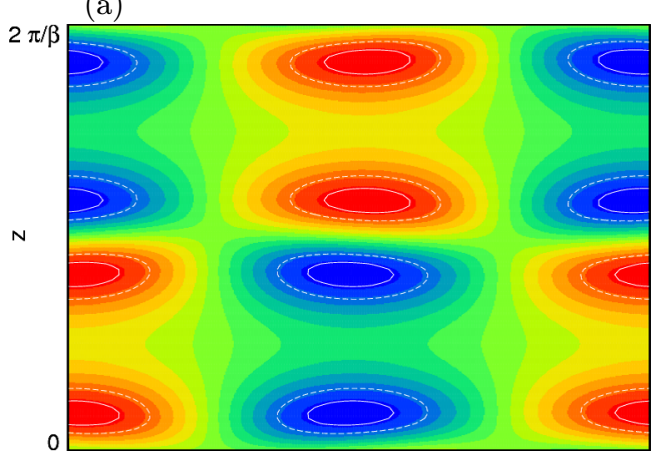

(c)

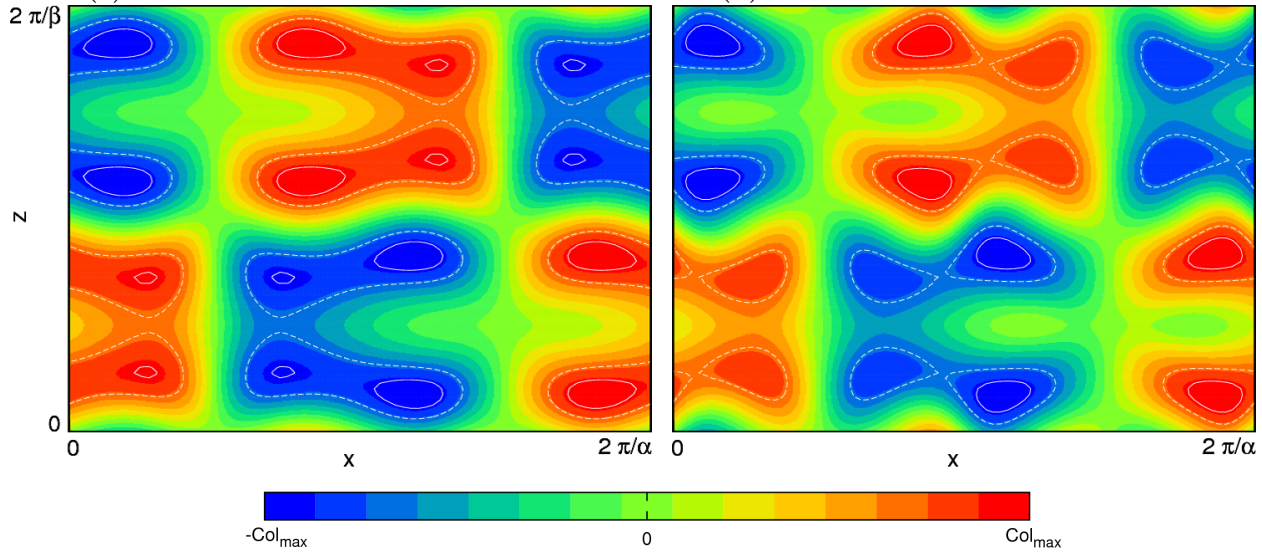

(b)

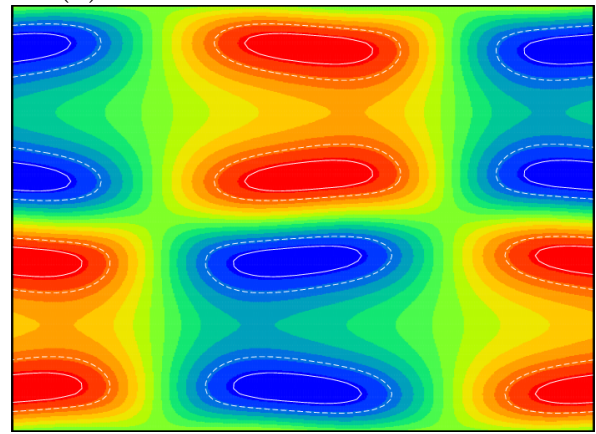

(d)

Figure 8. The streamwise velocity field $u$ at the mid-gap $y=0$. The solid and dashed white curves are iso-contours of $\pm 90 \%$ and $\pm 70 \% \mathrm{Col}_{\max }$ respectively. (a) $(\alpha, \beta)=(500,1.37)$, $\mathrm{Col}_{\max }=0.045 ;$ (b) $(\alpha, \beta)=(300,1.37), \mathrm{Col}_{\max }=0.059 ;(\mathrm{c})(\alpha, \beta)=(100,1.37)$, $\mathrm{Col}_{\max }=0.165 ;(\mathrm{d})(\alpha, \beta)=(71,1.37), \mathrm{Col}_{\max }=0.332$. 
(a)

(b)
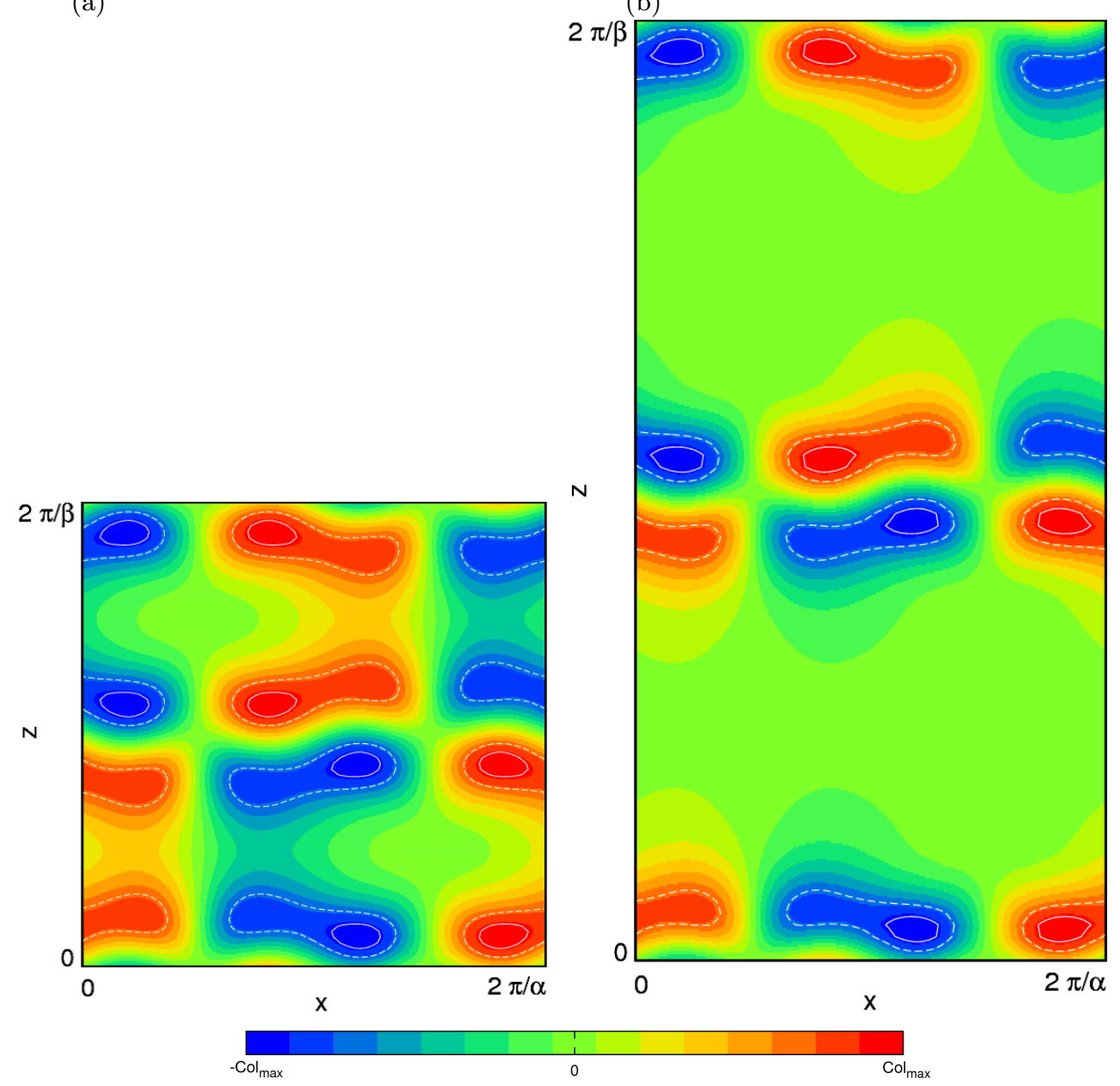

Figure 9. The streamwise velocity field $u$ at the mid-gap $y=0$. The solid and dashed white curves are iso-contours of $\pm 90 \%$ and $\pm 70 \% \mathrm{Col}_{\max }$ respectively. (a) $(\alpha, \beta)=(100,1)$, $\mathrm{Col}_{\max }=0.148 ;(\mathrm{b})(\alpha, \beta)=(100,0.5), \mathrm{Col}_{\max }=0.148$.

speculating of course is that, in the same way as fully turbulent flows apparently need some backbone of equilibrium structures on which to hang, flows which exhibit turbulent spots similarly need a backbone of solutions exhibiting localization on which to hang.

We note that periodic solutions, see for example Kawahara and Kida (2001), are also of huge importance in the turbulent dynamics of an evolving flow. Similarly the localized solutions we have found are likely to be just part of a number of solutions which may play some role in the evolution of turbulent spots.

For the Reynolds numbers at which Navier-Stokes simulations are possible, upper branch solutions are also visited and are thought to relate to upper bounds for the turbulence and dissipation (Gibson et al. 2008). It is not yet known whether upper branch modes occur at high values of $R$ since no equivalent theory to HS2 has been developed for them and finite Reynolds number calculations fail to follow them when $R$ is large. Thus one might argue about the relevance of our solutions to turbulent spots. However, by analogy with the recent discovery of homoclinic orbits of van Veen and Kawahara 
(a)

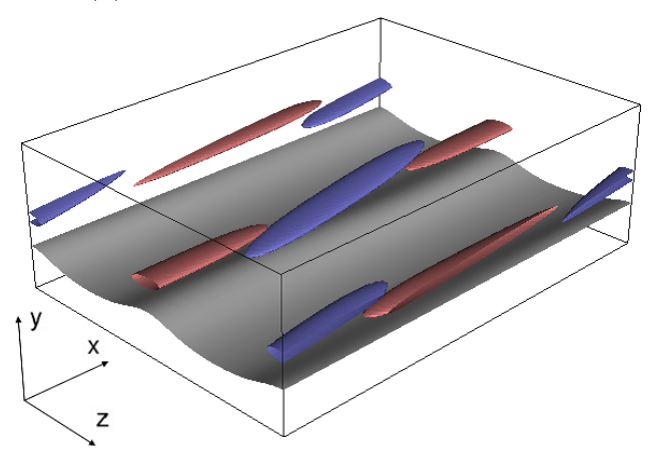

(b)

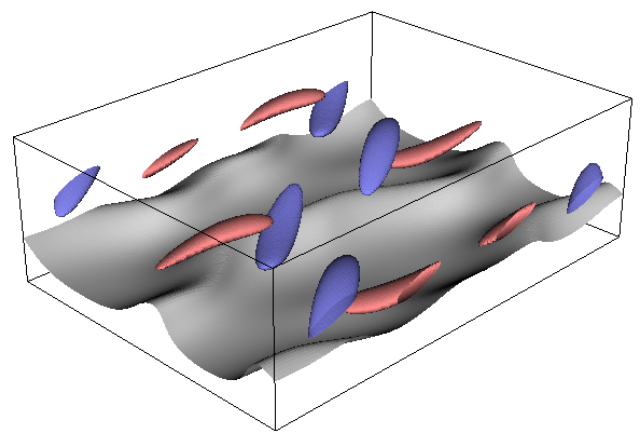

(c)

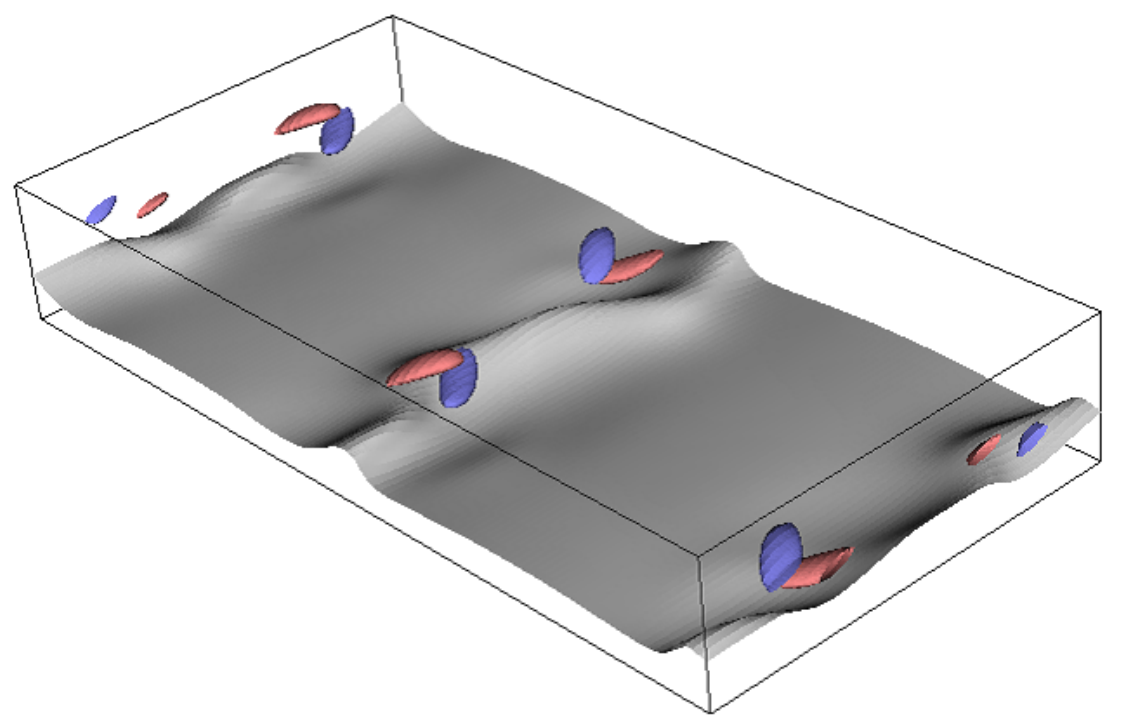

FiguRE 10. The grey surface represents an isosurface of $50 \%$ of the maximum magnitude of the streamwise velocity in the lower half of the channel. The red/blue bubbles are the isosurface of $80 \%$ of the maximum/minimum of the streamwise vorticity. (a) $(\alpha, \beta)=(500,1.37)$; (b) $(\alpha, \beta)=(71,1.37) ;(\mathrm{c})(\alpha, \beta)=(60,0.5)$.

(2011) in plane Couette flow, we can expect orbits homoclinic to our lower branch state which can mimic the localized burst of a turbulent spot.

Figure 11 shows further properties of the localized solutions but this time using the normal velocity component in the plane $y=0$. The parameter values in Figures 11a-c correspond to those of figure 10, and once again demonstrate the simultaneous spatial localization of the velocity field in both directions and if we concentrate on the most active regions we see alternating spots (denoted by dark red or blue) aligned along the centre of the channel. Given that the length of the channel has been scaled with the Reynolds number our results are quite consistent with the description given by Duguet et al. (2009) of elliptical-shaped spots of length increasing with Reynolds number; see for example Figure 2 of that paper. Thus it is possible that the edge states possibly associated with turbulent spots found by Duguet et al. (2009) are related to the localized equilibrium structures we have found. 
(a)
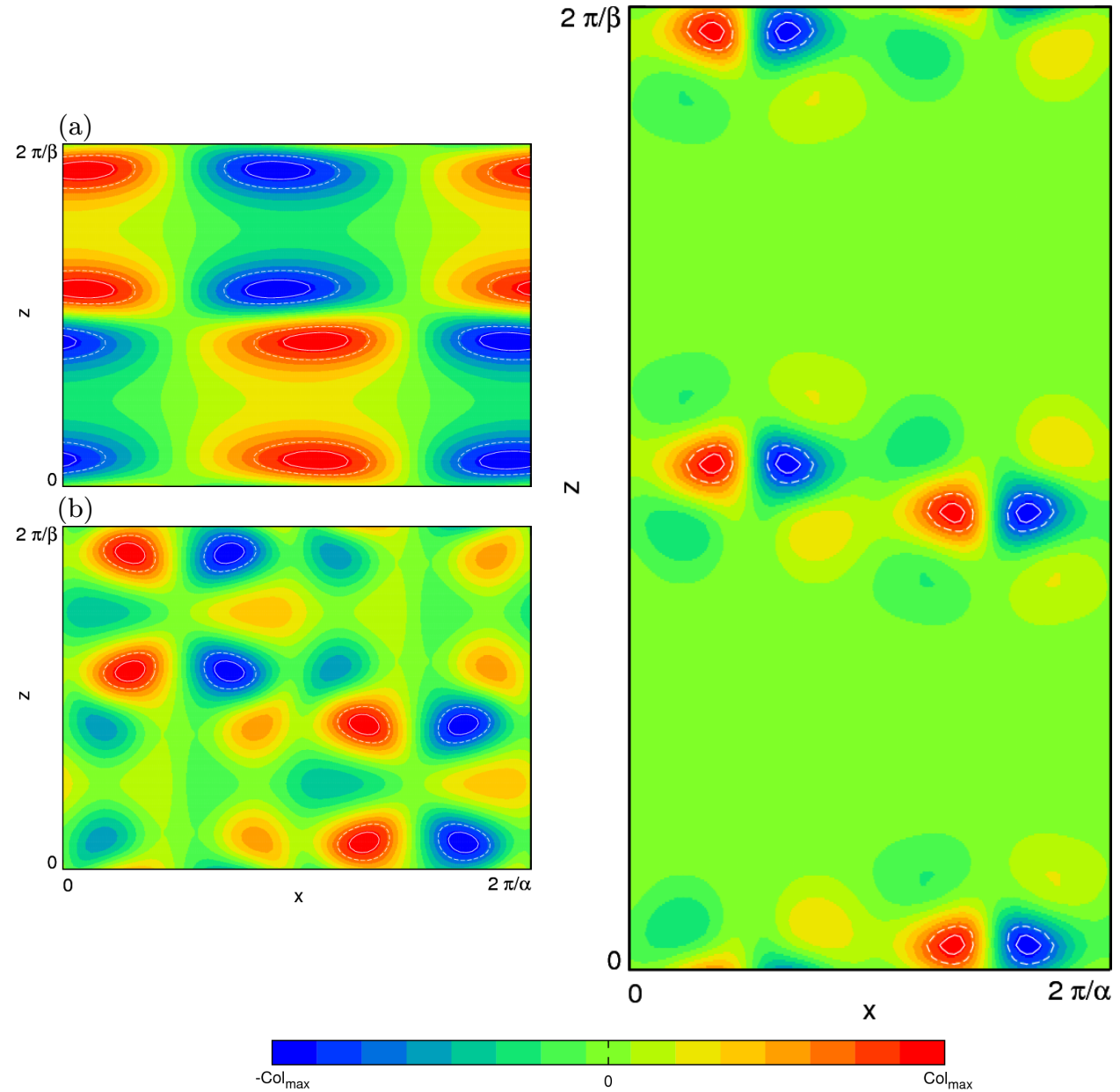

FIGURE 11. The wall-normal velocity field $v$ at the mid-gap $y=0$. The solid and dashed white curves are iso-contours of $\pm 90 \%$ and $\pm 70 \%$ of $\mathrm{Col}_{\max }$ respectively. (a) $(\alpha, \beta)=(500,1.37)$, $\mathrm{Col}_{\max }=2.10 ;(\mathrm{b})(\alpha, \beta)=(71,1.37), \mathrm{Col}_{\max }=17.09 ;(\mathrm{c})(\alpha, \beta)=(60,0.5), \mathrm{Col}_{\max }=15.19$.

Figure 12 shows similar results using a slightly different representation and now showing the effect at Reynolds numbers of 500 and 1000. The flow structure illustrated here is not unlike that shown in, for example, Figure 5 of Schneider et al. (2010a) and Figure 3 of Duguet et al. (2009). Further evidence that the equilibrium structures we have found are related to the edge states computed from the Navier-Stokes equations can be found by consideration of the energy of the flow. Figure 5 of Schneider et al. (2010a) plots the average kinetic energy of the flow. The averages are taken over the $y-z$ or $x-y$ planes and it was found that the localization in $z$ corresponds to 'double exponential' decay whilst the streamwise localization corresponds to just exponential decay. In Figure 13 we plot the average in $x-y$ of the energy in the streamwise velocity component $\left(u-u_{b}\right)^{2}$, and the energy associated with the flow in the $y-z$ plane, $v^{2}+w^{2}$. Note that since the velocity components in the $y-z$ plane have been scaled with $1 / R$ the energy of the flow is dominated by the streamwise velocity component (in the energy plots, these velocity components are divided by $\alpha$ instead). We see that when $\beta$ decreases from 1.0 to 0.5 the energy in the streamwise direction becomes localized in a manner consistent with Figure 5c of Schneider et al. (2010a). Interestingly this localization is already present 
(a)

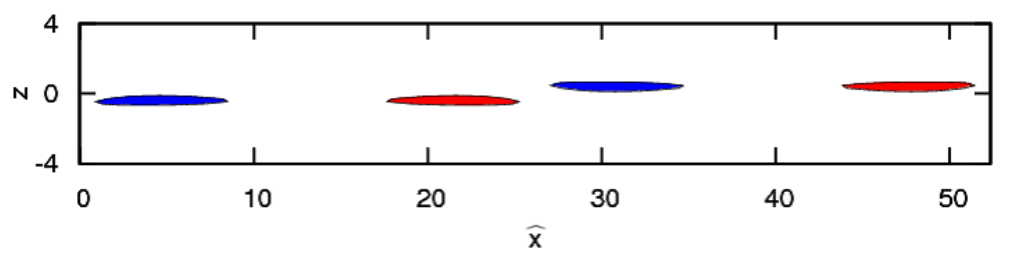

(b)

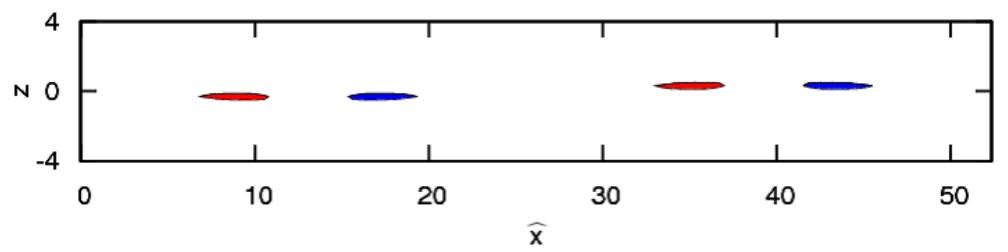

(c)

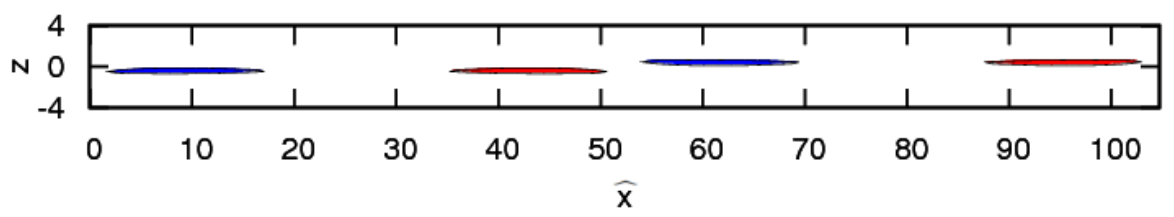

(d)

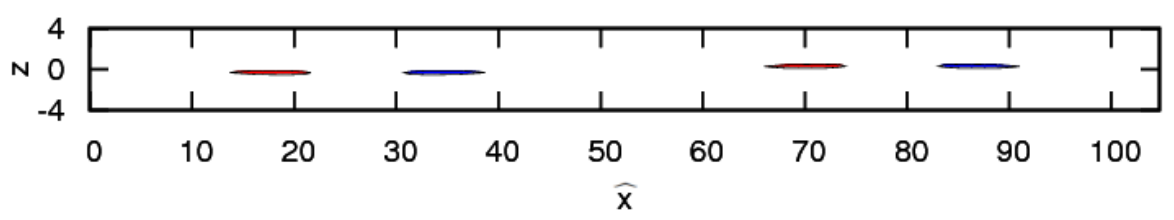

Figure 12. Red/blue represent the regions where the velocity exceeds $80 \%$ of its in-plane maximum/minimum. (a) Streamwise component $u$ on $y=0$; (b) wall-normal component $v$ on $y=0$. The solution at $(\alpha, \beta)=(60,0.5)$ is used. The streamwise coordinate is rescaled to the original variable $\widehat{x}$ by using $R=500$. (c) \& (d): same as (a) \& (b) but for $R=1000$.

when $\beta=1.0$ for the $v-w$ flow in the $y-z$ plane. Figure 14 shows similar results when the averages are taken in the $y-z$ plane. Once again we observe localization when $\alpha$ changes from 500 to 71 . The first of these plots shows similar behaviour to Figure $5 \mathrm{~b}$ of Schneider et al. (2010a) with the localization in $x$ not as pronounced as that observed in the spanwise direction. These results suggests very strongly that the edge states found from the unsteady Navier-Stokes equations have a very similar form to the equilibrium states found here.

Figure 15a shows the mean (over both $x$ and $z$ ) streamwise velocity component at different values of $\alpha$ with $\beta=1.37$. We observe that for the higher values of $\alpha$, the meanflow correction changes sign within $[0,1]$ whereas as the localization develops, the mean flow correction is always of one sign. However as the streamwise wavenumber decreases the mean-flow correction becomes S-shaped. Figure 15b shows the mean-flow correction for a fixed $\alpha=100$ at different values of $\beta$. The results suggest strongly that the mean-flow correction in the limit $\beta \rightarrow 0$ is proportional to $\beta S(y)$, where $S(y)$ is close to the solid line in that figure. For any $\beta$, this limiting profile, when added to the basic state, has an inflexion point at the origin so is itself unstable to inviscid waves of wavelength comparable with the channel half-depth $h$. Thus it is not surprising that the localization 
(a)

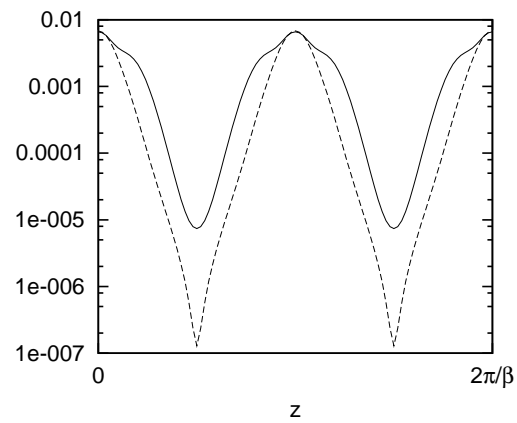

(b)

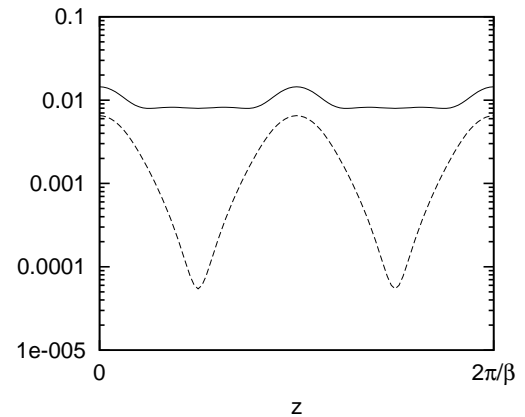

FIGURE 13. Averages in $x-y$ of the energy associated with the perturbation. The solid line shows the energy associated with the streamwise velocity, $\left(u-u_{b}\right)^{2}$, while the dashed line shows the energy associated with the flow in the $y-z$ plane, $v^{2}+w^{2}$, divided by $\alpha^{2}$. (a) $(\alpha, \beta)=(100,0.5)$, (b) $(\alpha, \beta)=(100,1.0)$.

(c)

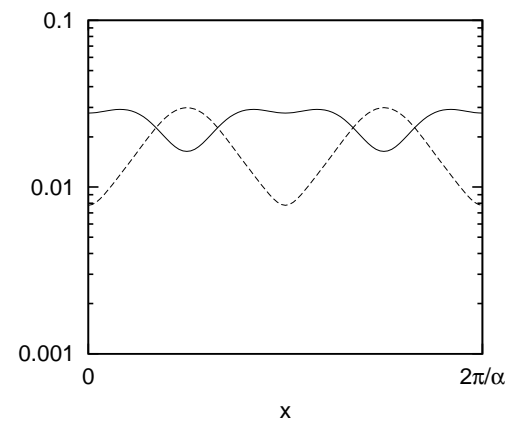

(d)

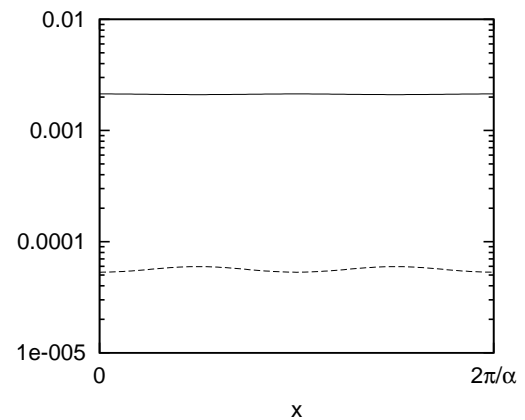

FiguRE 14. Averages in $y-z$ of the energy associated with the perturbation. The solid line shows the energy associated with the streamwise velocity, $\left(u-u_{b}\right)^{2}$, while the dashed line shows the energy associated with the flow in the $y-z$ plane, $v^{2}+w^{2}$, divided by $\alpha^{2}$. (a) $(\alpha, \beta)=(71,1.37)$, (b) $(\alpha, \beta)=(500,1.37)$.

develops when the higher harmonics corresponding to wavelengths comparable with $h$ in the streamwise direction grow. However, the implication of the canonical scaling for the mean-flow correction and the relation to fully-turbulent mean profiles, whilst intriguing, is not pursued here. In fact the limiting form of the mean-flow correction agrees well with turbulent mean profiles for plane Couette flow; see for example Figure 2 of Komminaho et al. (1996). It appears then that the basic shape for the mean flow correction in a turbulent boundary layer is driven by the large scale structures described here.

Now let us turn to a discussion of the significance of the critical value of $\alpha$ at which the equilibrium structures we calculate develop spatial localization. We assume that the value of $\alpha \simeq 70$ where the $\beta=1.0,1.37$ solutions localize is in fact the limit as $\beta \rightarrow 0$ of the values of $\alpha$ where localization develops for a given $\beta$. We saw earlier that as this develops the energy cascades down into the higher harmonics and formally our analysis will fail when there is $O(1)$ energy in the harmonics of order $R$. At that stage our original assumption used to derive our interaction equations fails and, at the very least locally, the full Navier-Stokes equations must be solved and the full detailed spot structures found by Duguet et al. (2009) and Schneider et al. (2010a) cannot be found without doing so. However the existence of a critical wavenumber for a given spanwise wavelength at which short scale structures can develop predicts a critical minimum box length needed 
(a):

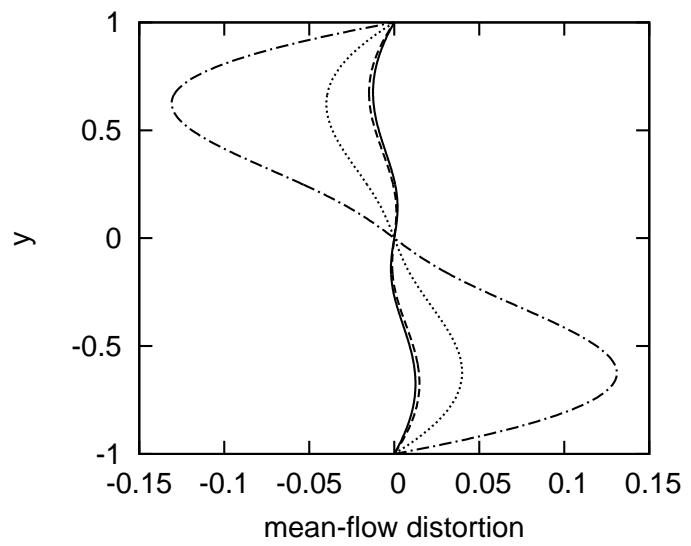

(b):

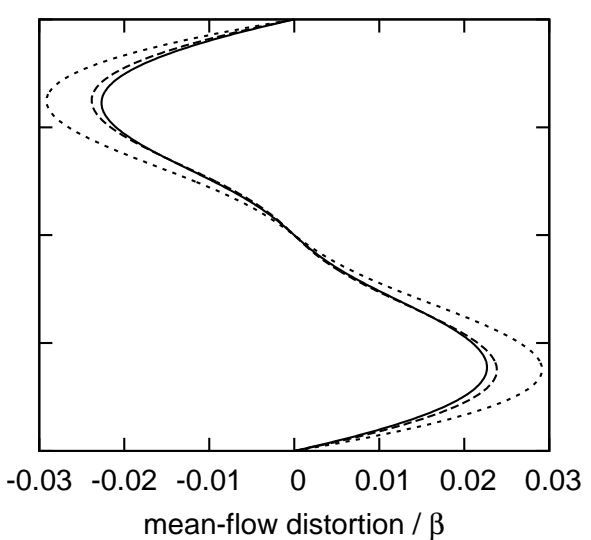

Figure 15. Mean-flow distortion $\left(\bar{u}-u_{b}\right)$. (a) the dash-dotted, dotted, dashed and solid curves are $(\alpha, \beta)=(71,1.37),(\alpha, \beta)=(100,1.37),(\alpha, \beta)=(300,1.37)$ and $(\alpha, \beta)=(500,1.37)$; (b) the dotted, dashed and solid curves are $(\alpha, \beta)=(100,1.37),(\alpha, \beta)=(100,1)$ and $(\alpha, \beta)=(100,0.5)$.

for localized structures possibly related to turbulent spots to form. The existence of such a minimum length for spot formation has been known by the computational community for some time; see for example Lundbladh and Johansson (1991), Komminaho et al. (1996). If the result we find is indeed a predictor of the box length needed to allow spot formation to begin it is significant because for any high Reynolds number it tells us how long the computational box used must be in order to capture turbulent spots. In that case it can be argued that VWI predicts successfully two of the key results found by computational experimentation regarding turbulent flows. Firstly the upper $O(1)$ streamwise wavenumber limit predicted in HS2 for VWI states to exist at high $R$ apparently is related to the minimum box size needed to sustain turbulent flow. This would infer that fully turbulent flows can occur only if a backbone of equilibrium states such as those described by VWI exist. Secondly the present calculation clearly shows that equilibrium localized states only develop when the streamwise wavenumber decreases to a critical value. This corresponds to the box length increasing to a critical value proportional to the Reynolds number before localization can occur. If the localization is indeed the embryonic stage of a turbulent spot then we have predicted the minimum box length needed for spot formation. The discussion in Lundbladh and Johansson (1991) and indeed in Duguet et al. (2009) is certainly consistent with that notion.

This work was partially supported by the JSPS Institutional Program for Young Researcher Overseas Visits. K.D. thanks Prof. M. Nagata and Dr D. P. Wall for helpful discussions, and all the authors would like to thank the referees for their insightful comments. 


\section{Appendix A. The energy stability limit for the long-wave interaction equations}

Here we apply standard energy analysis arguments (e.g. Joseph and Carmi 1969) to the long-wave interaction equations (2.11) in order to derive a bound on the scaled wavenumber $\alpha$, below which all disturbances must monotonically decay.

First we write $\mathbf{u}=(y+\widetilde{u}, \widetilde{v}, \widetilde{w}), p=\widetilde{p}$ and substitute into (2.11). The fluctuations are then governed by

$$
\begin{aligned}
\frac{D \widetilde{\mathbf{u}}}{D \tau}+\left(y \widetilde{u}_{x}+\widetilde{v}, y \widetilde{v}_{x}, y \widetilde{w}_{x}\right) & =-\left(0, \widetilde{p}_{y}, \widetilde{p}_{z}\right)+\left(\partial_{y y}^{2}+\partial_{z z}^{2}\right) \widetilde{\mathbf{u}}, \\
\nabla \cdot \widetilde{\mathbf{u}} & =0,
\end{aligned}
$$

and are subject to zero boundary conditions on $y= \pm 1$ and periodic boundary conditions in $x, z$ with wavenumbers $\alpha, \beta$ respectively. If we consider a periodic box we can derive the equivalent of the Reynolds-Orr energy equation in the form

$$
\frac{D E}{D \tau}=I_{1}-I_{2}
$$

where we have defined

$$
E=\frac{1}{2}\left\langle\widetilde{u}^{2}\right\rangle, \quad I_{1}=-\langle\widetilde{u} \widetilde{v}\rangle, I_{2}=\left\langle\widetilde{u}_{y}^{2}+\widetilde{u}_{z}^{2}\right\rangle>0,
$$

with $\langle *\rangle$ the volumetric average operator defined in section 3. It follows that if $D E / D \tau>$ 0 over some interval in $\tau$ then we require $M=\max m>1$, where $m=I_{1} / I_{2}$. Suppose that the solution which maximizes $m$ is $\widetilde{\mathbf{u}}_{0}=\left(\widetilde{u}_{0}, \widetilde{v}_{0}\right)$. We then consider all other solutions in the form $(\widetilde{u}, \widetilde{v})=\widetilde{\mathbf{u}}_{0}+\varepsilon\left(\widetilde{u}_{1}, \widetilde{v}_{1}\right)$ with corresponding energy ratio $m(\varepsilon)$. By definition $M=m(0)$ and $m^{\prime}(0)=0$. Since $m(\varepsilon) I_{2}(\varepsilon)=I_{1}(\varepsilon)$, we have

$$
M=I_{1}^{\prime}(0) / I_{2}^{\prime}(0) \text {. }
$$

It's readily established that

$$
I_{1}^{\prime}(0)=-\left\langle\widetilde{\mathbf{u}}_{1} \cdot\left(\widetilde{v}_{0} \mathbf{e}_{x}+\widetilde{u}_{0} \mathbf{e}_{y}\right)\right\rangle, \quad I_{2}^{\prime}(0)=-2\left\langle\widetilde{\mathbf{u}}_{1} \cdot\left(\widetilde{u}_{0 y y}+\widetilde{u}_{0 z z}\right) \mathbf{e}_{x}\right\rangle,
$$

and hence

$$
\left\langle\widetilde{\mathbf{u}}_{1} \cdot\left\{2 M\left(\widetilde{u}_{0 y y}+\widetilde{u}_{0 z z}\right) \mathbf{e}_{x}-\left(\widetilde{v}_{0} \mathbf{e}_{x}+\widetilde{u}_{0} \mathbf{e}_{y}\right)\right\}\right\rangle=0,
$$

for arbitrary solenoidal fields $\widetilde{\mathbf{u}}_{1}$. It follows that the expression in \{\} can be written in potential form $\nabla \phi$, and thus the maximizing state is governed by

$$
-\widetilde{v}_{0}+2 M\left(\widetilde{u}_{0 y y}+\widetilde{u}_{0 z z}\right)=\phi_{x},-\widetilde{u}_{0}=\phi_{y}, \quad 0=\phi_{z},
$$

together with $\nabla \cdot \widetilde{\mathbf{u}}_{0}=0$. The solution of the energy problem which maximizes $m$ is therefore two-dimensional (in contrast to our computed solutions of (2.11) which are, of course, strongly three-dimensional).

Introducing a streamfunction $\psi(y) \exp (\mathrm{i} \alpha x)$ to satisfy continuity, we find that

$$
\psi^{\prime \prime \prime \prime}+\frac{\mathrm{i} \alpha}{M} \psi^{\prime}=0, \quad \psi=\psi^{\prime}=0 \text { on } y= \pm 1 .
$$

Integration and application of the boundary conditions leads to the eigenvalue equation

$$
\left(1+e^{-2 q \sqrt{3}}\right) \cos (q)-2 e^{-q \sqrt{3}} \cos (2 q)-\sqrt{3}\left(1-e^{-2 q \sqrt{3}}\right) \sin (q)=0,
$$

for $q$, where $q^{3}=\alpha / M$. Since we require $M>1$ for energy growth we need $\alpha>q_{0}^{3}$ where $q_{0}$ is the smallest positive root of (A 1). From computation $q_{0} \simeq 3.666$, and hence the 
lower bound on $\alpha$ is given by

$$
\alpha>q_{0}^{3} \simeq 49.272 .
$$

As remarked in the main text, the full numerical simulations of (2.11) generate results for values of $\alpha$ that are only slightly in excess of this number, indicating that this bound is surprisingly sharp.

\section{REFERENCES}

Benney, D. 1984 The evolution of disturbances in shear flows at high Reynolds numbers. Stud. Appl. Math. 70, 1-19.

Chen, C. S. \& Kuo, W. J. 2004 Heat transfer characteristics of gaseous flow in long mini- and microtubes, Numer. Heat Transfer, Part A: 46, 497-514.

Clever, R. M. \& Busse, F. H. 1997 Tertiary and quaternary solutions for plane Couette flow. J. Fluid Mech. 344, 137-153.

Duguet, Y., Schlatter P. \& Henningson, D. S. 2009 Localized edge states in plane Couette flow. Phys. Fluids 21, 111701.

Faisst, H. \& Eckhardt, B. 2003 Traveling waves in pipe flow. Phys. Rev. Lett. 91, 224502.

Fletcher, C. A. J. 1991 Computational Techniques for Fluid Dynamics, vol. 2. Springer.

Gibson, J. F., Halcrow, J. \& Cvitanovic, P. 2008. Visualizing the geometry of state space in plane Couette flow. J. Fluid Mech. 611, 107-130.

Gibson, J. F., Halcrow, J. \& Cvitanovic, P. 2009. Equilibrium and travelling-wave solutions of plane Couette flow. J. Fluid Mech. 638, 1-24.

Gittler, Ph. 1993 Stability of axial Poiseuille-Couette flow between concentric cylinders. Acta Mech. 101, 1-13.

Goldstein, M. E. \& Wundrow, D. W. 1998 On the environmental realizability of algebraically growing disturbances and their relation to Klebanoff modes. Theo. Comp. Fluid Dyn. 10, $171-186$.

Goldstein, M. E. \& Sescu, A. 2008 Boundary-layer transition at high free-stream disturbance levels - beyond Klebanoff modes. J. Fluid Mech. 613, 95-124.

Hall, P. 1988 The nonlinear development of Gortler vortices in growing boundary layers. J. Fluid Mech. 193, 243-266.

Hall, P. 2012a Vortex-wave interactions/Self-sustained processes in high Prandtl number natural convection in a vertical channel with moving sidewalls. Stud. Appl. Math. 129, 1-25.

Hall, P. 2012b Vortex-wave interactions: long wavelength streaks and spatial localisation in natural convection. J. Fluid Mech. In Press.

Hall, P. \& Horseman, N. J. 1991 The linear inviscid secondary instability of longitudinal vortex structures in boundary layers. J. Fluid Mech. 232, 357-375.

Hall, P. \& Sherwin, S. 2010 Streamwise vortices in shear flows: harbingers of transition and the skeleton of coherent structures. J. Fluid Mech. 661, 178-205.

Hall, P. \& Smith, F. T. 1989 Nonlinear Tollmien-Schlichting/vortex interaction in boundary layers. Eur. J.Mech. B/Fluids 8 (3), 179-205.

Hall, P. \& Smith, F. T. 1990 Near Planar TS Waves and Longitudinal Vortices in Channel Flow: Nonlinear Interaction and Focussing, pp. 5-39. Springer.

Hall, P. \& Smith, F. T. 1991 On strongly nonlinear vortex/wave interactions in boundary-layer transition. J. Fluid Mech. 227, 641-666.

Higuera, M. \& Vega, J. 2009 Modal description of internal optimal streaks. J. Fluid Mech. 626, $21-31$.

Itano, T. \& Generalis, S. C. 2009 Hairpin vortex solution in planar Couette flow: a tapestry of knotted vortices. Phys. Rev. Lett. 102, 114501-114504.

Itano, T. \& Toh, S. 2001 The dynamics of bursting process in wall turbulence. J. Phys. Soc. Jpn. 70, 703-716.

Kawahara, G. \& Kida, S. 2001 Periodic motion embedded in plane Couette turbulence: regeneration cycle and burst. J. Fluid Mech. 449, 291-300.

Komminaho, K., Lundbladh, A. \& Johansson, A.V. 1996 Very large structures in plane turbulent Couette flow. J. Fluid Mech. 320, 259-285. 
Joseph, D. D. \& Carmi, S. 1969 Stability of Poiseuille flow in pipes, annuli, and channels. Quart. Applied Math. 26, 575-599.

Kerswell, R. R, \& Tutty, O. R. 2007 Recurrence of travelling waves in transitional pipe flow. J. Fluid Mech. 584, 69-102.

Luchini, P. 2000 Reynolds-number-independent instability of the boundary layer over a flat surface: optimal perturbations. J. Fluid Mech. 404, 289-309.

Lundbladh, A. \& Johansson, A. V. 1991 Direct simulation of turbulent spots in plane Couette flow. J. Fluid Mech. 229, 499-516.

Nagata, M. 1990 Three-dimensional finite-amplitude solutions in plane Couette flow: bifurcation from infinity. J. Fluid Mech. 217, 519-527.

Peris, R., Marquina, A. \& Candela, V. 2011 The convergence of the perturbed Newton method and its application for ill-conditioned problems. Appl. Math Comput. 218, 2988-3001.

Schneider, T.M., Marinc, D. \& Eckhardt, B 2010a Localised edge states nucleate turbulence in extended plane Couette cells, . J. Fluid Mech. 646, 441-451.

Schneider, T. M., Gibson, J. F. \& Burke, J. 2010b Snakes and ladders: localized solutions of plane Couette flow. Phys. Rev. Lett. 104, 104501.

Schneider, T. M., Gibson, J. F., Lagha, M., Lillo, F. De \& Eckhardt, B. 2008 Laminar-turbulent boundary in plane Couette flow. Phys. Rev. E, 78, 037301.

Skufca, J. D., Yorke, J. A. \& Eckhardt, B. 2006 Edge of chaos in a parallel shear flow. Phys. Rev. Lett. 96, 174101-174104.

Smith, F. T. 1979 Instability of flow through pipes of general cross-section. Part 1. Mathematika 26, $187-210$.

Smith, F. T. \& Bodonyi, R. J. 1980 On the stability of the developing flow in a channel or circular pipe. Q. J. Mech. Appl. Maths 33, 293-320.

Tillmark, N. \& Alfredsson, P. H. 1992. Experiments on transition in plane Couette flow. J. Fluid Mech. 235, 89-102.

Tillmark, N. 1995 On the spreading mechanisms of a turbulent spot in plane Couette flow. Europhys. Lett. 32, 481.

van Veen, L. \& Kawahara, G. 2011 Homoclinic tangle on the edge of shear turbulence. Phys. Rev. Lett. 107, 114501.

Viswanath, D. 2009 The critical layer in pipe flow at high Reynolds number. Phil. Trans. R. Soc. A367, 561-576.

Waleffe, F. 1995 Hydrodynamic stability and turbulence: beyond transients to a self-sustaining process. Stud. Appl. Math. 95, 319-343.

Waleffe, F. 1997 On a self-sustaining process in shear flows. Phys. Fluids 9, 883-900.

Waleffe, F. 1998 Three-dimensional coherent states in plane shear flows. Phys. Rev. Lett. 81, 4140-4143.

Waleffe, F. 2001 Exact coherent structures in channel flow. J. Fluid Mech. 435, 93-102.

Waleffe, F. 2003 Homotopy of exact coherent structures in plane shear flows. Phys. Fluids 15, $1517-1534$.

Walton, A. G. 2002 The temporal evolution of neutral modes in the impulsively started flow through a circular pipe and their connection to the nonlinear stability of Hagen-Poiseuille flow. J. Fluid Mech. 457, 339-376.

Walton, A. G. 2004 Stability of circular Poiseuille-Couette flow to axisymmetric disturbances. J. Fluid Mech. 500, 169-210.

Wang, J., Gibson, J. \& Waleffe, F. 2007 Lower branch coherent states in shear flows: transition and control. Phys. Rev. Lett. 98, 204501-204504.

Wedin, H. \& Kerswell, R. R. 2004 Exact coherent structures in pipe flow: travelling wave solutions. J. Fluid Mech. 508, 333-371.

Willis, A. P. \& Kerswell, R. R. 2009 Turbulent dynamics of pipe flow captured in a reduced model: puff relaminarisation and localised 'edge' states. J. Fluid Mech. 619, 213-233.

Zuccher, S., Tumin, A. \& Reshotko, E. 2006 Parabolic approach to optimal perturbations in compressible boundary layers. J. Fluid Mech. 556, 189-216. 